\title{
$\beta$-Catenin pathway activation in breast cancer is associated with triple-negative phenotype but not with CTNNB1 mutation
}

\author{
Felipe C Geyer ${ }^{1,4}$, Magali Lacroix-Triki ${ }^{1,2,4}$, Kay Savage ${ }^{1}$, Monica Arnedos ${ }^{3}$, \\ Maryou B Lambros ${ }^{1}$, Alan MacKay ${ }^{1}$, Rachael Natrajan ${ }^{1}$ and Jorge S Reis-Filho,1 \\ ${ }^{1}$ Molecular Pathology Laboratory, The Breakthrough Breast Cancer Research Centre, Institute of Cancer \\ Research, London, UK; ${ }^{2}$ Institut Claudius Regaud, Toulouse, France and ${ }^{3}$ Breast Unit, The Royal Marsden \\ Hospital, London, UK
}

\begin{abstract}
Aberrant $\beta$-catenin expression as determined by assessment of its subcellular localization constitutes a surrogate marker of Wnt signalling pathway activation and has been reported in a subset of breast cancers. The association of $\beta$-catenin/Wnt pathway activation with clinical outcome and the mechanisms leading to its activation in breast cancers still remain a matter of controversy. The aims of this study were to address the distribution of $\beta$-catenin expression in invasive breast cancers, the correlations between $\beta$-catenin expression and clinicopathological features and survival of breast cancer patients, and to determine whether aberrant $\beta$-catenin expression is driven by CTNNB1 ( $\beta$-catenin encoding gene) activating mutations. Immunohistochemistry was performed on a tissue microarray containing 245 invasive breast carcinomas from uniformly treated patients, using two anti- $\beta$-catenin monoclonal antibodies. Selected samples were subjected to CTNNB1 exon 3 mutation analysis by direct gene sequencing. A good correlation between the two $\beta$-catenin antibodies was observed (Spearman's $r>0.62, P<0.001$ ). Respectively, 31 and $11 \%$ of the cases displayed lack/reduction of $\beta$-catenin membranous expression and nuclear accumulation. Complete lack of $\beta$-catenin expression was significantly associated with invasive lobular carcinoma histological type. Subgroup analysis of non-lobular cancers or non-lobular grade 3 carcinomas revealed that lack/reduction of $\beta$-catenin membranous expression and/or nuclear accumulation were significantly associated with oestrogen receptor negativity, absence of $H E R 2$ gene amplification and overexpression, lack/reduction of E-cadherin expression and tumours of triple-negative and basal-like phenotype. Univariate survival analysis revealed a significant association between $\beta$-catenin nuclear expression and shorter metastasis-free and overall survival in the whole cohort; however, $\beta$-catenin nuclear expression was not an independent predictor of outcome in multivariate analysis. No CTNNB1 mutations were identified in the 28 selected breast carcinomas analysed. In conclusion, $\beta$-catenin/Wnt pathway activation is preferentially found in triple-negative/basal-like breast carcinomas, is associated with poor clinical outcome and is unlikely to be driven by CTNNB1 mutations in breast cancer.
\end{abstract}

Modern Pathology (2011) 24, 209-231; doi:10.1038/modpathol.2010.205; published online 12 November 2010

Keywords: breast cancer; immunohistochemistry; mutation; sequencing

$\beta$-Catenin is a multifunctional protein located to the intracellular side of the cytoplasmic membrane coded by the CTNNB1 gene, which maps to chromosome 3p22.1. It has a critical role in cell-to-

Correspondence: Professor JS Reis-Filho, MD, PhD, FRCPath, The Breakthrough Breast Cancer Research Centre, Institute of Cancer Research, 237 Fulham Road, London, SW3 6JB, UK.

E-mail: Jorge.Reis-Filho@icr.ac.uk

${ }^{4}$ These authors contributed equally to this work.

Received 23 July 2010; revised 23 August 2010; accepted 30

August 2010; published online 12 November 2010 cell adhesion by linking cadherins to the actin cytoskeleton and has a central role in transcriptional regulation in the Wnt signalling pathway. ${ }^{1}$ Indeed, upon Wnt activation, $\beta$-catenin is translocated from the membrane to the cytoplasm and nucleus, where it interacts with transcriptional activators to modulate a number of target genes associated with increased growth, invasion and cellular transformation, such as $C-M Y C^{2}$ or $c y c l i n D 1 .^{3,4}$

There are numerous lines of evidence to implicate the importance of $\beta$-catenin deregulation and 
CTNNB1 activating mutations in carcinogenesis. ${ }^{1,3-9}$ Several studies have addressed the issue of $\beta$-catenin/Wnt pathway dysfunction in breast cancer. ${ }^{3,10-17}$ Our previous study on spindle lesions of the breast demonstrated that aberrant $\beta$-catenin expression is often observed in metaplastic carcinomas of the breast, indicating that Wnt canonical pathway activation is found in at least a subset of breast cancers. ${ }^{18}$ Expression of $\beta$-catenin in breast cancer and its association with outcome have been a matter of controversy. Although some have reported that aberrant $\beta$-catenin expression is found in breast cancers of poor outcome $e^{3,13,15}$ and of the basal-like phenotype, ${ }^{19}$ others have failed to demonstrate a correlation between $\beta$-catenin aberrant expression and outcome. ${ }^{11,14,16,17,20}$ This is not surprising, given the difficulties in assessing $\beta$-catenin/Wnt pathway activation in breast cancer, because of the complexity of this molecular pathway, the challenging interpretation of $\beta$-catenin subcellular localization, specificity of different antibodies and other technical issues. ${ }^{12,20}$

The mechanisms underpinning $\beta$-catenin/Wnt pathway activation in breast cancer remain poorly understood. CTNNB1 activating mutations and $A P C$ inactivating mutations are some of the possible mechanisms leading to $\beta$-catenin nuclear accumulation. The prevalence of these molecular abnormalities is highly debated in breast cancer. ${ }^{21-24}$ Although some authors have described CTNNB1 mutations in up to $26 \%$ of metaplastic carcinomas of the breast ${ }^{25}$ we $^{18}$ and others ${ }^{7}$ have failed to confirm the presence of CTNNB1 mutations in this histological type of breast tumours. Notwithstanding the fact that most recent studies agree in that $\beta$-catenin/ Wnt pathway is activated in at least a subset of breast cancers, ${ }^{15,18,19}$ the molecular alterations underlying aberrant $\beta$-catenin expression are yet to be elucidated.

The aims of this study were threefold: (1) to address the distribution of $\beta$-catenin expression as defined by immunohistochemistry using two antibodies in a cohort of 245 invasive breast carcinomas uniformly treated with anthracycline-based chemotherapy, (2) to correlate $\beta$-catenin expression with clinicopathological characteristics and outcome of primary breast cancer patients and (3) to investigate whether activation of $\beta$-catenin/Wnt pathway in breast cancer is driven by CTNNB1 gene mutation.

\section{Materials and methods}

\section{Case Selection and Tissue Microarray}

A cohort of 245 patients with invasive breast cancer was included in a tissue microarray containing three replicate $0.6 \mathrm{~mm}$ cores. All patients were diagnosed and managed at the Royal Marsden Hospital, London, UK, between 1994 and 2000. All patients were primarily treated with surgery followed by anthracycline-based chemotherapy. Adjuvant endocrine therapy was prescribed for patients with oestrogen receptor-positive tumours (tamoxifen alone in $96 \%$ of the patients for the available follow-up period). Complete follow-up was available for 244 patients, ranging from 0.5 to 125 months (median $=67$ months, mean $=67$ months). Tumours were graded according to a modified Bloom-Richardson scoring system, ${ }^{26}$ and size was categorized according to the TNM staging. ${ }^{27}$ The study was approved by the Royal Marsden Hospital Ethics Committee.

\section{Immunohistochemistry}

Immunohistochemistry was performed on $3 \mu \mathrm{m}$ thick tissue microarray sections. For $\beta$-catenin immunohistochemistry, two commercially available monoclonal antibodies raised against the C-terminal domain of $\beta$-catenin were used, clone $14 / \beta$-catenin (BD Transduction Laboratories, San Jose, CA, USA) and 17C2 (Novocastra/Leica, Newcastle Upon Tyne, UK), which were used in 1:6000 and 1:100 dilutions, respectively, as previously described ${ }^{18}$ and summarized in Supplementary Table 1. Immunohistochemical analysis with the $14 / \beta$-catenin clone was performed with the observers blinded to the results of the analysis of $17 \mathrm{C} 2$ clone. Results of $\beta$-catenin immunohistochemistry obtained with each antibody were analysed independently by two of the authors (ML-T and FCG) using the Allred scoring system for cytoplasmic and nuclear reactivity. This scoring system combines the staining intensity and the percentage of stained cells (intensity score $0-3+$ $\%$ score 0-5). ${ }^{28}$ An Allred score of $>2$ was considered as positive. $\beta$-catenin membranous staining was scored according to a previously used system for E-cadherin. ${ }^{29}$ Briefly, the proportion of stained cells with complete membranous staining was recorded in four categories: $0,0-10 \%$; 1 , $10-<25 \% ; 2,25-<50 \% ; 3, \quad 50-75 \%$; and 4 , $>75 \%$. Expression of $\beta$-catenin was considered normal when scores were $\geq 3$, reduced when equal to 2 , and negative when scores were $<2$.

$\beta$-Catenin expression was correlated with the expression of oestrogen receptor, progesterone receptor, HER2, epidermal growth factor receptor (EGFR), cytokeratin (CK) 14, CK5/6 and CK17, Ki67, p53, topoisomerase II $\alpha$ (TOP2A), caveolin-1 (CAV1), caveolin-2 (CAV2), FOXA1, E-cadherin, CD44, Bcl2, nestin and cyclin D1, and with amplification of CCND1, HER2, TOP2A and MYC. Details of the methods and results of the above proteins are described elsewhere ${ }^{30,31}$ and summarized in Supplementary Table 1 . The prevalence of CCND1, HER2, TOP2A and MYC gene amplification was assessed by chromogenic in situ hybridization with SpotLight CISH probes (Invitrogen, Paisley, UK) and results not in relation to $\beta$-catenin expression were reported elsewhere. ${ }^{32,33}$ Based upon the expression of HER2, oestrogen receptor, CK5/6 and EGFR, 
tumours were classified into basal, HER2 and luminal according to the immunohistochemical panel proposed by Nielsen et al. ${ }^{34}$

\section{Microdissection and DNA Extraction}

Cases with $\beta$-catenin nuclear expression and/or of triple-negative phenotype (ie, oestrogen receptor-, progesterone receptor- and HER2-negative) from the tissue microarray were selected for CTNNB1 sequencing analysis based on an Allred score for $\beta$-catenin nuclear staining $=2(n=9)$ or $>2(n=19)$. All cases were microdissected to ensure $>75 \%$ of purity of neoplastic cells. Microdissection of formalin-fixed paraffin-embedded samples was performed with a sterile needle under a stereomicroscope (Olympus SZ61, Tokyo, Japan) from ten consecutive $8 \mu \mathrm{m}$ thick sections stained with nuclear fast red as previously described. ${ }^{35}$ DNA was extracted using the DNeasy Kit (Qiagen, Crawley, UK) according to the manufacturer's recommendations. DNA concentration was measured with the PicoGreen ${ }^{-}$assay as per the manufacturer's instructions (Invitrogen). ${ }^{35}$ Out of the 30 carcinomas selected for the CTNNB1 mutation analysis, microdissection yielded sufficient DNA of optimal quality in 28 samples.

\section{CTNNB1 Mutation Analysis}

Sequencing of known mutation hotspots of CTNNB1 on exon $3^{5,9,21,23,25}$ was performed in 19 invasive carcinomas of the breast displaying $\beta$-catenin nuclear expression, and 9 cases with a $\beta$-catenin nuclear Allred score of 2 (ie, considered as negative) and of triple-negative phenotype. As previously described, ${ }^{18}$ positive controls (ie, DNA samples of the HCT116 colon cancer cell line ${ }^{36}$ and of one formalinfixed paraffin-embedded breast fibromatosis, which harbored an exon 3 CTNNB1 mutation) were included in each experiment. The primers used for CTNNB1 sequencing were previously described. ${ }^{25} \mathrm{~A}$ total of $50 \mathrm{ng}$ tumour DNA was amplified and sequencing reactions were carried out using the DNA Sequencing Kit BigDye Terminator v 1.1 Cycle Sequencing Ready Reaction Mix (Applied Biosystems, Warrington, UK) as previously described. ${ }^{36}$ Sequences of the forward and reverse strands were analysed with Mutation Surveyor software (Softgenetics, State College, PA, USA). All reactions were carried out in duplicate from the original DNA sample.

\section{Statistical Analysis}

Data analysis of $\beta$-catenin expression was performed with the results obtained with each antibody. The SPSS statistical software package was used for all statistical analysis. Spearman's correlations coefficient and unweighted $\kappa$ scores were assessed to determine the concordance between results obtained with the two anti- $\beta$-catenin antibodies used in this study. For each parameter, correlations between categorical variables were performed using the $\chi^{2}$ test and Fisher's exact test. Metastasis-free survival was expressed as the number of months from diagnosis to the occurrence of distant relapse. Disease-free survival was expressed as the number of months from diagnosis to the occurrence of distant, local relapse or death (disease-related death). Overall survival was expressed as the number of months from diagnosis to the occurrence of breast-cancer related death. Cumulative survival probabilities were calculated using the KaplanMeier method. Differences between survival rates were tested with the log-rank test. A $P$-value of $\leq 0.05$ was considered as statistically significant.

\section{Results}

\section{$\beta$-Catenin Expression in Invasive Breast Cancer}

The results of the immunohistochemical analysis of $\beta$-catenin expression are summarized in Table 1. Owing to lost/fragmented cores or cores without invasive tumour on tissue microarray sections, $\beta$-catenin expression data with both antibody clones were available in 221 out of 245 tumours. As previously described, ${ }^{18}$ a good correlation between the two commercially available antibodies raised against $\beta$-catenin, clones $14 / \beta$-catenin and $17 \mathrm{C} 2$, was found. We observed statistically significant correlations between both antibodies for the semiquantitative assessment of $\beta$-catenin membranous (Spearman's $\quad r=0.863, \quad P<0.0001$ ), cytoplasmic (Spearman's $r=0.620, P<0.0001$ ), and nuclear expression (Spearman's $r=0.676, P<0.0001$ ) (Table 1). Analysis of agreement between the two antibodies revealed a substantial to nearly perfect agreement, with $\kappa$ scores of $0.801,0.893$ and 0.672 for membranous (negative vs reduced vs normal), cytoplasmic (positive vs negative) and nuclear (positive vs negative) reactivity, respectively.

In the 221 cases with available data for $14 / \beta$ catenin clone, $\beta$-catenin membranous expression was normal in $152(69 \%)$ cases, reduced in $19(9 \%)$ cases and negative in $50(22 \%)$ cases. $\beta$-catenin cytoplasmic and nuclear expression (Allred score $>2$ ) was observed in 195 (88\%) and $25(11 \%)$ tumours, respectively (Table 1). A weak cytoplasmic $\beta$-catenin expression was found in the neoplastic cells (ie, Allred score $>2$ ) in most of the tumours, rendering the objective interpretation of $\beta$-catenin expression in this subcellular compartment challenging. Therefore, although cytosolic accumulation of $\beta$-catenin is considered to be an aberrant form of $\beta$-catenin expression and, potentially, indicative of Wnt pathway activation, ${ }^{19,25}$ in this study, aberrant expression was defined as lack/reduction of $\beta$-catenin membrane expression and/or $\beta$-catenin nuclear expression $(n=83,37 \%)$. Of the total of 
69 cases displaying lack/reduction of $\beta$-catenin membrane expression, 11 also showed nuclear accumulation.

Table 1 Comparison of $\beta$-catenin immunostainings in 221 invasive carcinomas of the breast using two commercially available antibodies (clones 14/ $\beta$-catenin and 17C2)

\begin{tabular}{|c|c|c|}
\hline & $\begin{array}{c}\text { Clone } 14 / \beta \text {-catenin } \\
\mathrm{n}=221\end{array}$ & $\begin{array}{c}\text { Clone 17C2 } \\
\mathrm{n}=221\end{array}$ \\
\hline \multicolumn{3}{|c|}{$\beta$-Catenin membranous expression } \\
\hline \multicolumn{3}{|l|}{ Reduction score } \\
\hline 0 & $35(15.8 \%)$ & $33(15 \%)$ \\
\hline 1 & $15(6.8 \%)$ & $15(6.8 \%)$ \\
\hline 2 & $19(8.6 \%)$ & $24(10.8 \%)$ \\
\hline 3 & $42(19 \%)$ & $39(17.6 \%)$ \\
\hline 4 & $110(49.8 \%)$ & $110(49.8 \%)$ \\
\hline Spearman's $r$, two tailed & \multicolumn{2}{|c|}{$0.863(P<0.0001)$} \\
\hline \multicolumn{3}{|c|}{$\beta$-Catenin cytoplasmic expression } \\
\hline \multicolumn{3}{|c|}{ Allred score } \\
\hline 0 & $24(10.9 \%)$ & $23(10.4 \%)$ \\
\hline 2 & $2(0.9 \%)$ & $4(1.8 \%)$ \\
\hline 3 & $6(2.7 \%)$ & $11(5 \%)$ \\
\hline 4 & $19(8.6 \%)$ & $24(10.9 \%)$ \\
\hline 5 & $53(24 \%)$ & $100(45.2 \%)$ \\
\hline 6 & $105(47.5 \%)$ & $53(24 \%)$ \\
\hline 7 & $11(5 \%)$ & $5(2.3 \%)$ \\
\hline 8 & $1(0.4 \%)$ & $1(0.4 \%)$ \\
\hline Spearman's $r$, two tailed & \multicolumn{2}{|c|}{$0.620(P<0.0001)$} \\
\hline \multicolumn{3}{|l|}{$\beta$-Catenin nuclear expression } \\
\hline \multicolumn{3}{|l|}{ Allred score } \\
\hline 0 & $174(78.7 \%)$ & $198(89.6 \%)$ \\
\hline 2 & $22(10 \%)$ & $8(3.6 \%)$ \\
\hline 3 & $14(6.4 \%)$ & $6(2.7 \%)$ \\
\hline 4 & $5(2.3 \%)$ & $3(1.4 \%)$ \\
\hline 5 & $1(0.4 \%)$ & $3(1.4 \%)$ \\
\hline 6 & $1(0.4 \%)$ & $2(0.9 \%)$ \\
\hline 7 & $3(1.4 \%)$ & 0 \\
\hline 8 & $1(0.4 \%)$ & $1(0.4 \%)$ \\
\hline Spearman's $r$, two tailed & \multicolumn{2}{|c|}{$0.676(P<0.0001)$} \\
\hline
\end{tabular}

\section{$\beta$-Catenin Pattern of Expression Differs According to} the Histological Types of Breast Cancer

$\beta$-Catenin expression was significantly correlated with histological type (Table 2 and Figure 1). The majority of invasive ductal carcinomas, in particular those of low histological grade, displayed a normal pattern of $\beta$-catenin expression, that is, normal membranous expression (81\%) without nuclear localization (88\%; Figures 2a and b); whereas, in a way akin to E-cadherin expression in lobular carcinomas of the breast, ${ }^{29}$ a strong correlation between lack of $\beta$-catenin membranous expression and lobular histological type was found (82\%, $P<0.001$, Figures 1a, c, 2c and d). Likewise, the vast majority of lobular carcinomas did not display cytoplasmic and nuclear expression of $\beta$-catenin (Table 2, Figure 2d and Supplementary Table 2). Furthermore, reduced or negative membranous expression $(40 \%)$ and positive nuclear expression $(30 \%)$ were more prevalent in carcinomas of other histological types (Figure 1), such as in a metaplastic carcinoma (Figures 2e and f). It is noteworthy that this is a pattern frequently observed in this subtype of breast cancer. ${ }^{18}$

\section{$\beta$-Catenin Aberrant Expression Correlates with Triple-Negative and Basal-Like Phenotypes}

$\beta$-Catenin expression in the distinct subcellular compartments was correlated with clinicopathological parameters in the whole cohort. The results with $14 / \beta$-catenin clone in relation to membranous and nuclear $\beta$-catenin expression are summarized in Table 3. Aberrant $\beta$-catenin expression (ie, lack/ reduction of membranous expression and/or nuclear expression) was significantly correlated with histo-

Table $2 \beta$-Catenin membranous and nuclear expression in 222 invasive breast carcinomas according to histological type

14/ $\beta$-catenin clone

\begin{tabular}{|c|c|c|c|c|c|c|c|c|}
\hline \multirow[b]{2}{*}{ Туре } & \multicolumn{4}{|c|}{ Membranous expression } & \multicolumn{4}{|c|}{ Nuclear expression } \\
\hline & $\mathrm{N}=222$ & Negative & Reduced & Positive & $\mathrm{P}$-value & Negative & Positive & $\mathrm{P}$-value \\
\hline IDC & & 16 & 15 & 129 & & 140 & 20 & \\
\hline ILC & & 28 & 1 & 5 & $<0.001$ & 32 & 2 & 0.091 \\
\hline Mixed & & 4 & 2 & 12 & & 18 & 0 & \\
\hline Other & & 3 & 1 & 6 & & 7 & 3 & \\
\hline
\end{tabular}

17C2 clone

\begin{tabular}{|c|c|c|c|c|c|c|c|c|}
\hline \multirow[b]{2}{*}{ Type } & \multicolumn{4}{|c|}{ Membranous expression } & \multicolumn{4}{|c|}{ Nuclear expression } \\
\hline & $\mathrm{N}=222$ & Negative & Reduced & Positive & $\mathrm{P}$-value & Negative & Positive & $\mathrm{P}$-value \\
\hline IDC & & 17 & 17 & 127 & & 149 & 12 & \\
\hline ILC & & 25 & 4 & 5 & $<0.001$ & 34 & 0 & $<0.001$ \\
\hline Mixed & & 3 & 3 & 12 & & 18 & 0 & \\
\hline Other & & 3 & 0 & 6 & & 6 & 3 & \\
\hline
\end{tabular}

IDC: invasive ductal carcinoma; ILC: invasive lobular carcinoma. 


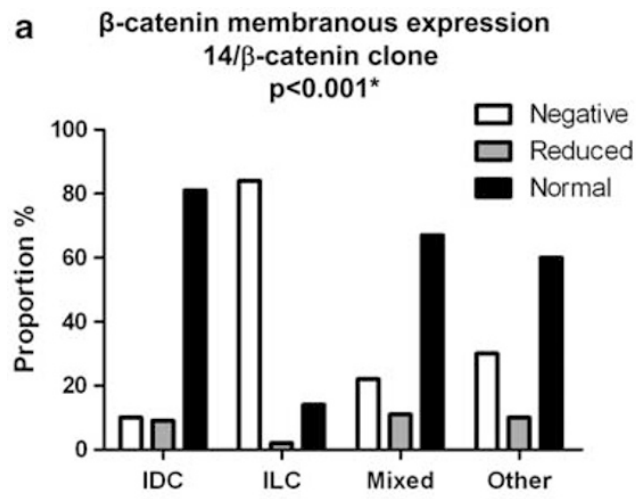
b $\quad \beta$-catenin nuclear expression $14 / \beta$-catenin clone $\mathrm{p}=0.091^{\star}$
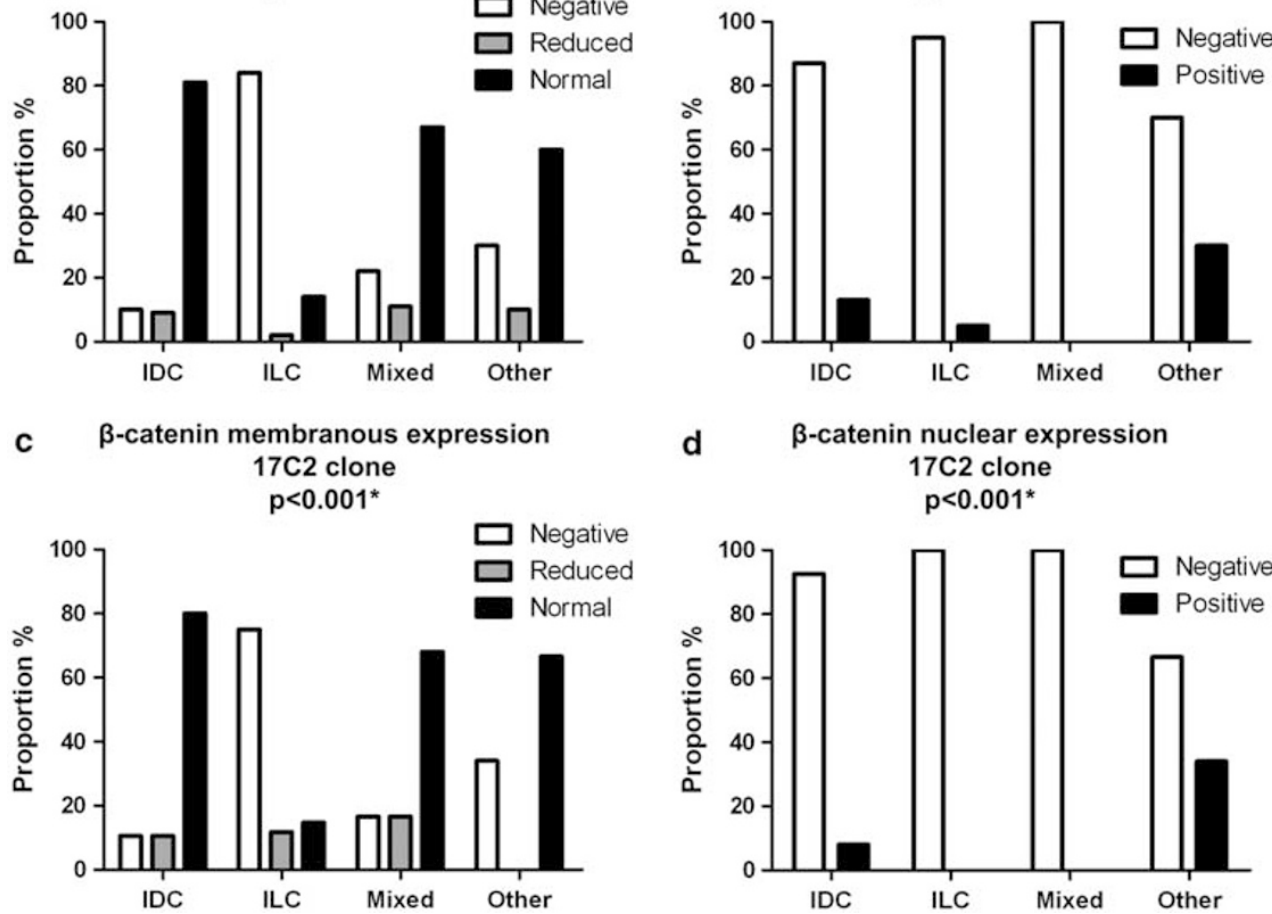

d $\beta$-catenin nuclear expression
$17 \mathrm{C} 2$ clone
$\mathrm{p}<0.001^{*}$

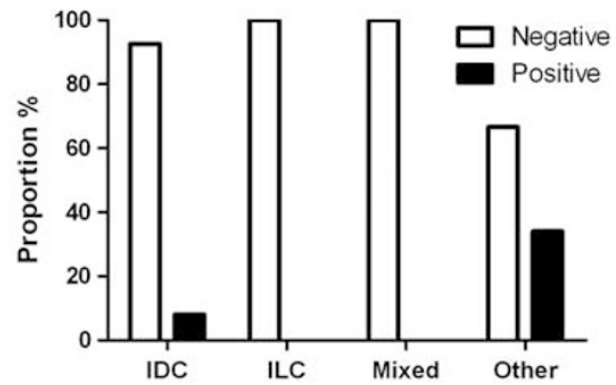

Figure $1 \beta$-Catenin expression in invasive breast carcinomas according to histological type. $\beta$-Catenin membranous and nuclear expression using $14 / \beta$-catenin clone $(\mathbf{a}, \mathbf{b})$ and $17 \mathrm{C} 2$ clone $(\mathbf{c}, \mathbf{d})$ according to distinct histological type in the whole cohort $(n=222)$. ${ }^{*} \chi^{2}$ test.

logical grade, presence of lympho-vascular invasion and lymph node metastasis, triple negativity (ie, oestrogen receptor-, progesterone receptor- and HER2-negative), basal-like phenotype and basal-like features, as described below $(P<0.05$, Table 3$)$. As expected, given that the great majority of lobular carcinomas displayed complete lack of $\beta$-catenin expression, a significant correlation between E-cadherin and $\beta$-catenin membranous expression was found $(P<0.001$, Table 3$)$. However, $\beta$-catenin nuclear expression was also associated with lack/ reduction of E-cadherin in the whole cohort $(P=0.027$, Table 3$)$, indicating that the association between lack/reduction of both E-cadherin and aberrant $\beta$-catenin expression may be due not only to the lobular histology. Analysis of $\beta$-catenin expression with the clone 17C2 revealed similar associations (Table 4). Owing to the subjective nature of the analysis of $\beta$-catenin cytoplasmic expression, it is not surprising that no associations between $\beta$-catenin cytoplasmic expression and clinicopathological parameters were observed (Supplementary Table 2).

To avoid the confounding factor of the distinctive pattern of $\beta$-catenin expression in lobular carcinomas, $\beta$-catenin expression in the distinct subcellular compartments was investigated after the exclusion of all lobular carcinomas $(n=186$ cases). Associations similar to those observed in the analysis of $\beta$-catenin expression in the whole cohort were found (Tables 5 and 6, and Supplementary Table 2). Using $14 / \beta$ catenin clone, a significant inverse correlation between aberrant (reduced/negative membranous and/ or positive nuclear expression) $\beta$-catenin expression and oestrogen receptor, oestrogen receptor pathwayassociated parameters, such as progesterone receptor, FOXA1, cyclin D1 and Bcl2, and HER2 gene amplification and overexpression was found $(P<0.05$, Table 5). Furthermore, a positive correlation was observed between aberrant $\beta$-catenin expression and expression of EGFR, basal CKs (CK5/6, CK14, CK17) and other markers typically found in basal-like breast carcinomas, ${ }^{34,37-40}$ such as p53 positivity, high proliferation indices as defined by MIB-1 expression, expression of CAV1, CAV2 and nestin $(P<0.05$, Table $5)$. Not surprisingly, aberrant $\beta$-catenin expression was significantly associated with triple-negative and basal-like phenotypes (Table 5 and Figures 3a, b and 4). It is noteworthy that despite the exclusion of lobular carcinomas, the association between reduction/lack of E-cadherin expression and both lack/ reduction of $\beta$-catenin membranous staining and nuclear $\beta$-catenin accumulation was still significant (Table 5). Similar observations were obtained when tumours were analysed with the 17C2 clone (Table 6 and Figures 3c and d).

As the vast majority of basal-like and triplenegative tumours are of histological grade 3 , one 

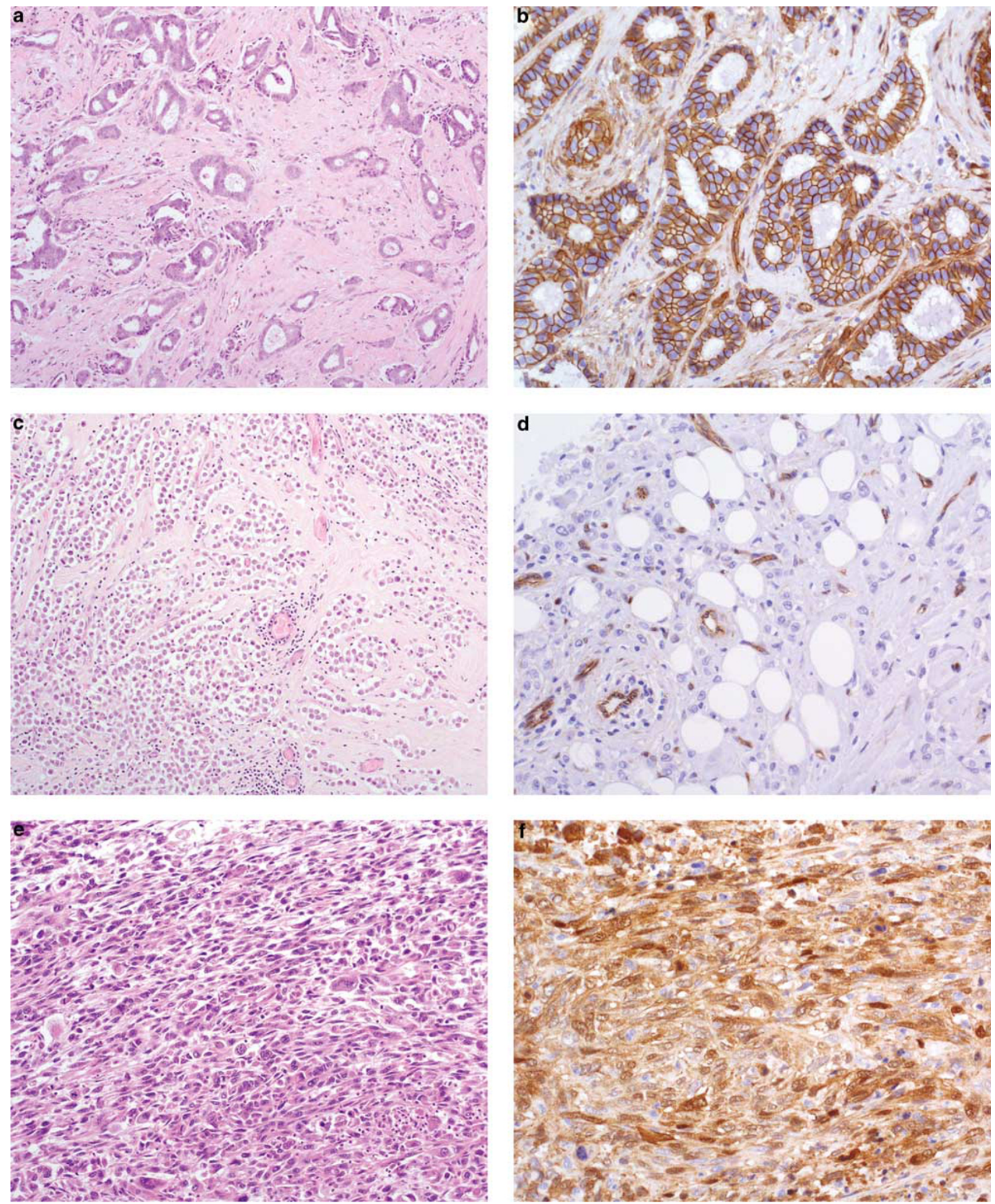

Figure $2 \beta$-Catenin expression in invasive breast carcinomas according to histological type. The vast majority ( $>80 \%)$ of invasive ductal carcinomas (a, b) displayed normal $\beta$-catenin membranous expression and lacked $\beta$-catenin nuclear expression, whereas invasive lobular carcinomas lacked any $\beta$-catenin expression (c, d). Lack/reduction of $\beta$-catenin membranous expression and positive nuclear expression were more prevalent in carcinomas of other histological subtype, such as metaplastic carcinomas (e, f). 
Table $3 \beta$-Catenin membranous and nuclear expression in 222 invasive breast carcinomas (14/ $\beta$-catenin clone)

\begin{tabular}{|c|c|c|c|c|c|c|c|c|}
\hline Parameter & $\mathrm{N}$ & $\begin{array}{l}\text { Membranous } \\
\text { negative }\end{array}$ & $\begin{array}{l}\text { Membranous } \\
\text { reduced }\end{array}$ & $\begin{array}{l}\text { Membranous } \\
\text { positive }\end{array}$ & P-value & $\begin{array}{l}\text { Nuclear } \\
\text { negative }\end{array}$ & $\begin{array}{l}\text { Nuclear } \\
\text { positive }\end{array}$ & $\mathrm{P}$-value \\
\hline Size-TNM & 221 & & & & 0.4679 & & & 0.108 \\
\hline $\mathrm{T} 1$ & & 24 & 7 & 83 & & 106 & 8 & \\
\hline $\mathrm{T} 2$ & & 21 & 11 & 60 & & 77 & 15 & \\
\hline T3 & & 5 & 1 & 9 & & 13 & 2 & \\
\hline Grade & 217 & & & & 0.1706 & & & 0.029 \\
\hline 1 & & 3 & 1 & 19 & & 23 & 0 & \\
\hline 2 & & 19 & 3 & 40 & & 58 & 4 & \\
\hline 3 & & 27 & 15 & 90 & & 111 & 21 & \\
\hline$L V I$ & 220 & & & & 0.0428 & & & 0.374 \\
\hline Negative & & 23 & 7 & 41 & & 61 & 10 & \\
\hline Positive & & 27 & 12 & 110 & & 134 & 15 & \\
\hline$L N$ mets & 215 & & & & 0.9061 & & & 0.044 \\
\hline Negative & & 18 & 6 & 48 & & 59 & 13 & \\
\hline Positive & & 32 & 13 & 98 & & 131 & 12 & \\
\hline$E R$ & 222 & & & & 0.0432 & & & $<\mathbf{0 . 0 0 1}$ \\
\hline Negative & & 11 & 7 & 22 & & 25 & 15 & \\
\hline Positive & & 40 & 12 & 130 & & 172 & 10 & \\
\hline$P R$ & 222 & & & & 0.091 & & & $<\mathbf{0 . 0 0 1}$ \\
\hline Negative & & 19 & 7 & 35 & & 46 & 15 & \\
\hline Positive & & 32 & 12 & 117 & & 151 & 10 & \\
\hline HER2 & 222 & & & & 0.0424 & & & 0.03 \\
\hline Negative & & 47 & 13 & 130 & & 165 & 25 & \\
\hline Positive & & 4 & 6 & 22 & & 32 & 0 & \\
\hline HER2 CISH & 211 & & & & 0.0442 & & & 0.029 \\
\hline Not amp & & 47 & 13 & 120 & & 156 & 24 & \\
\hline Amp & & 4 & 6 & 21 & & 31 & 0 & \\
\hline$E G F R$ & 222 & & & & 0.513 & & & 0.003 \\
\hline Negative & & 46 & 16 & 140 & & 184 & 18 & \\
\hline Positive & & 5 & 3 & 12 & & 13 & 7 & \\
\hline CK14 & 220 & & & & 0.0671 & & & $<0.001$ \\
\hline Negative & & 45 & 15 & 142 & & 187 & 15 & \\
\hline Positive & & 5 & 4 & 9 & & 8 & 10 & \\
\hline CK5/6 & 213 & & & & 0.8458 & & & $<\mathbf{0 . 0 0 1}$ \\
\hline Negative & & 43 & 15 & 133 & & 178 & 13 & \\
\hline Positive & & 6 & 2 & 14 & & 11 & 11 & \\
\hline CK17 & 219 & & & & 0.0601 & & & $<\mathbf{0 . 0 0 1}$ \\
\hline Negative & & 43 & 14 & 137 & & 178 & 16 & \\
\hline Positive & & 7 & 5 & 13 & & 16 & 9 & \\
\hline Basal CKs & 220 & & & & 0.214 & & & $<0.001$ \\
\hline Negative & & 42 & 14 & 133 & & 176 & 13 & \\
\hline Positive & & 8 & 5 & 18 & & 19 & 12 & \\
\hline $\begin{array}{l}\text { Basal CKs or } \\
\text { EGFR }\end{array}$ & 220 & & & & 0.0921 & & & $<\mathbf{0 . 0 0 1}$ \\
\hline Negative & & 40 & 13 & 131 & & 174 & 10 & \\
\hline Positive & & 10 & 6 & 20 & & 21 & 15 & \\
\hline P53 & 208 & & & & 0.0206 & & & 0.007 \\
\hline Negative & & 35 & 8 & 106 & & 138 & 11 & \\
\hline Positive & & 15 & 10 & 34 & & 46 & 13 & \\
\hline$M I B-1$ & & & & & 0.3661 & & & $<\mathbf{0 . 0 0 1}$ \\
\hline$<10 \%$ & & 20 & 4 & 60 & & 78 & 6 & \\
\hline $10-30 \%$ & & 23 & 9 & 61 & & 85 & 8 & \\
\hline$>30 \%$ & & 7 & 5 & 18 & & 19 & 11 & \\
\hline
\end{tabular}


Table 3 Continued

\begin{tabular}{|c|c|c|c|c|c|c|c|c|}
\hline Parameter & $\mathrm{N}$ & $\begin{array}{l}\text { Membranous } \\
\text { negative }\end{array}$ & $\begin{array}{l}\text { Membranous } \\
\text { reduced }\end{array}$ & $\begin{array}{c}\text { Membranous } \\
\text { positive }\end{array}$ & P-value & $\begin{array}{l}\text { Nuclear } \\
\text { negative }\end{array}$ & $\begin{array}{l}\text { Nuclear } \\
\text { positive }\end{array}$ & $\mathrm{P}$-value \\
\hline $\begin{array}{l}\text { Molecular } \\
\text { subtype }\end{array}$ & 216 & & & & 0.0042 & & & $<\mathbf{0 . 0 0 1}$ \\
\hline Basal & & 8 & 5 & 14 & & 14 & 13 & \\
\hline HER2 & & 4 & 6 & 23 & & 33 & 0 & \\
\hline Luminal & & 37 & 6 & 113 & & 146 & 10 & \\
\hline Triple-negative & 218 & & & & 0.0072 & & & $<\mathbf{0 . 0 0 1}$ \\
\hline No & & 41 & 13 & 135 & & 178 & 11 & \\
\hline Yes & & 10 & 6 & 13 & & 15 & 14 & \\
\hline E-cadherin & 201 & & & & $<\mathbf{0 . 0 0 1}$ & & & 0.027 \\
\hline Negative & & 33 & 6 & 19 & & 49 & 9 & \\
\hline Reduced & & 2 & 2 & 10 & & 12 & 2 & \\
\hline Normal & & 12 & 10 & 107 & & 118 & 11 & \\
\hline ТОР $2 A$ & 196 & & & & 0.3746 & & & 0.373 \\
\hline Low & & 17 & 8 & 65 & & 82 & 8 & \\
\hline High & & 29 & 9 & 68 & & 92 & 14 & \\
\hline TOP2A CISH & 211 & & & & 0.0142 & & & 0.234 \\
\hline Not amp & & 47 & 14 & 132 & & 169 & 24 & \\
\hline Amp & & 3 & 5 & 10 & & 18 & 0 & \\
\hline Cyclin D1 & 208 & & & & 0.0646 & & & 0.002 \\
\hline Low & & 11 & 3 & 10 & & 17 & 7 & \\
\hline Intermediate & & 9 & 3 & 30 & & 35 & 7 & \\
\hline High & & 29 & 13 & 100 & & 133 & 9 & \\
\hline CCND1 CISH & 221 & & & & 0.4839 & & & 0.542 \\
\hline Not amp & & 43 & 15 & 133 & & 168 & 23 & \\
\hline Amp & & 8 & 4 & 18 & & 28 & 2 & \\
\hline$M Y C \mathrm{CISH}$ & 184 & & & & 0.1075 & & & 0.418 \\
\hline Not amp & & 40 & 12 & 115 & & 149 & 18 & \\
\hline Amp & & 1 & 3 & 13 & & 14 & 3 & \\
\hline Caveolin 1 & 222 & & & & 0.2008 & & & $<\mathbf{0 . 0 0 1}$ \\
\hline Negative & & 43 & 17 & 141 & & 185 & 16 & \\
\hline Positive & & 8 & 2 & 11 & & 12 & 9 & \\
\hline Caveolin 2 & 196 & & & & 0.3686 & & & $<\mathbf{0 . 0 0 1}$ \\
\hline Negative & & 43 & 16 & 125 & & 169 & 15 & \\
\hline Positive & & 1 & 2 & 9 & & 5 & 7 & \\
\hline Nestin & 166 & & & & 0.0751 & & & $<\mathbf{0 . 0 0 1}$ \\
\hline Negative & & 31 & 10 & 106 & & 136 & 11 & \\
\hline Positive & & 5 & 4 & 10 & & 10 & 9 & \\
\hline FOXA1 & 175 & & & & 0.0303 & & & $<\mathbf{0 . 0 0 1}$ \\
\hline Negative & & 13 & 7 & 23 & & 31 & 12 & \\
\hline Positive & & 31 & 7 & 94 & & 125 & 7 & \\
\hline Bcl2 & 172 & & & & 0.0891 & & & 0.027 \\
\hline Negative & & 15 & 9 & 37 & & 51 & 10 & \\
\hline Positive & & 24 & 6 & 80 & & 104 & 6 & \\
\hline
\end{tabular}

amp: amplified; CISH: chromogenic in situ hybridization; ER: oestrogen receptor; IDC: invasive ductal carcinoma; LN mets: lymph node metastasis; LVI: lympho-vascular invasion; PR: progesterone receptor.

${ }^{\mathrm{a}}$ Molecular subtypes as defined by Nielsen immunohistochemical surrogate panel. ${ }^{34}$

Significant $P$-values are shown in bold.

could argue that the above correlations would be a mere reflection of the associations between high histological grade and aberrant $\beta$-catenin expression. However, subgroup analysis after the exclusion of grade 1 and 2 cancers and lobular carcinomas still revealed significant correlations between $\beta$-catenin aberrant expression and triplenegative and basal-like phenotypes, as well as with markers characteristically expressed by basal-like breast cancers, regardless of the antibody used 
Table $4 \beta$-Catenin membranous and nuclear expression in 222 invasive breast carcinomas (17C2 clone)

\begin{tabular}{|c|c|c|c|c|c|c|c|c|}
\hline Parameter & $\mathrm{N}$ & $\begin{array}{c}\text { Membranous } \\
\text { negative }\end{array}$ & $\begin{array}{l}\text { Membranous } \\
\text { reduced }\end{array}$ & $\begin{array}{l}\text { Membranous } \\
\text { positive }\end{array}$ & $\mathrm{P}$-value & $\begin{array}{l}\text { Nuclear } \\
\text { negative }\end{array}$ & $\begin{array}{l}\text { Nuclear } \\
\text { positive }\end{array}$ & P-value \\
\hline Size-TNM & 221 & & & & 0.300 & & & 0.917 \\
\hline $\mathrm{T} 1$ & & 22 & 9 & 83 & & 107 & 7 & \\
\hline $\mathrm{T} 2$ & & 20 & 14 & 58 & & 85 & 7 & \\
\hline T3 & & 5 & 1 & 9 & & 14 & 1 & \\
\hline Grade & 217 & & & & 0.426 & & & 0.203 \\
\hline 1 & & 2 & 2 & 19 & & 23 & 0 & \\
\hline 2 & & 17 & 6 & 40 & & 60 & 3 & \\
\hline 3 & & 27 & 15 & 89 & & 119 & 12 & \\
\hline$L V I$ & 220 & & & & 0.012 & & & 0.570 \\
\hline Negative & & 23 & 9 & 39 & & 65 & 6 & \\
\hline Positive & & 24 & 15 & 110 & & 140 & 9 & \\
\hline$L N$ mets & 215 & & & & 0.514 & & & 0.009 \\
\hline Negative & & 14 & 10 & 48 & & 62 & 10 & \\
\hline Positive & & 33 & 13 & 97 & & 138 & 5 & \\
\hline$E R$ & 222 & & & & 0.074 & & & $<\mathbf{0 . 0 0 1}$ \\
\hline Negative & & 14 & 4 & 22 & & 30 & 10 & \\
\hline Positive & & 34 & 20 & 128 & & 177 & 5 & \\
\hline$P R$ & 222 & & & & 0.017 & & & 0.001 \\
\hline Negative & & 21 & 5 & 35 & & 51 & 10 & \\
\hline Positive & & 27 & 19 & 115 & & 156 & 5 & \\
\hline HER2 & 222 & & & & 0.485 & & & 0.138 \\
\hline Negative & & 43 & 19 & 129 & & 176 & 15 & \\
\hline Positive & & 5 & 5 & 21 & & 31 & 0 & \\
\hline HER2 CISH & 211 & & & & 0.236 & & & 0.228 \\
\hline Not amp & & 43 & 18 & 120 & & 167 & 14 & \\
\hline Amp & & 5 & 6 & 19 & & 30 & 0 & \\
\hline EGFR & 222 & & & & 0.719 & & & 0.034 \\
\hline Negative & & 43 & 21 & 138 & & 191 & 11 & \\
\hline Positive & & 5 & 3 & 12 & & 16 & 4 & \\
\hline CK14 & 220 & & & & 0.244 & & & $<\mathbf{0 . 0 0 1}$ \\
\hline Negative & & 41 & 21 & 140 & & 193 & 9 & \\
\hline Positive & & 6 & 3 & 9 & & 12 & 6 & \\
\hline CK5/6 & 214 & & & & 0.894 & & & 0.002 \\
\hline Negative & & 40 & 21 & 131 & & 183 & 9 & \\
\hline Positive & & 5 & 3 & 14 & & 16 & 6 & \\
\hline$C K 17$ & 219 & & & & 0.159 & & & 0.076 \\
\hline Negative & & 40 & 19 & 135 & & 183 & 11 & \\
\hline Positive & & 7 & 5 & 13 & & 21 & 4 & \\
\hline Basal CKs & 220 & & & & 0.421 & & & 0.002 \\
\hline Negative & & 39 & 19 & 131 & & 181 & 8 & \\
\hline Positive & & 8 & 5 & 18 & & 24 & 7 & \\
\hline Basal CKs or EGFR & 220 & & & & 0.224 & & & $<\mathbf{0 . 0 0 1}$ \\
\hline Negative & & 36 & 19 & 129 & & 178 & 6 & \\
\hline Positive & & 11 & 5 & 20 & & 27 & 9 & \\
\hline P53 & 208 & & & & 0.218 & & & 0.027 \\
\hline Negative & & 30 & 15 & 104 & & 143 & 6 & \\
\hline Positive & & 18 & 7 & 34 & & 51 & 8 & \\
\hline MIB-1 & 207 & & & & 0.788 & & & 0.001 \\
\hline$<10 \%$ & & 16 & 10 & 59 & & 81 & 4 & \\
\hline $10-30 \%$ & & 22 & 10 & 60 & & 88 & 4 & \\
\hline$>30 \%$ & & 9 & 3 & 18 & & 23 & 7 & \\
\hline
\end{tabular}


Table 4 Continued

\begin{tabular}{|c|c|c|c|c|c|c|c|c|}
\hline Parameter & $\mathrm{N}$ & $\begin{array}{c}\text { Membranous } \\
\text { negative }\end{array}$ & $\begin{array}{l}\text { Membranous } \\
\text { reduced }\end{array}$ & $\begin{array}{c}\text { Membranous } \\
\text { positive }\end{array}$ & $\mathrm{P}$-value & $\begin{array}{l}\text { Nuclear } \\
\text { negative }\end{array}$ & $\begin{array}{l}\text { Nuclear } \\
\text { positive }\end{array}$ & $\mathrm{P}$-value \\
\hline Molecular subtype & 216 & & & & 0.096 & & & $<\mathbf{0 . 0 0 1}$ \\
\hline Basal & & 10 & 3 & 14 & & 19 & 8 & \\
\hline HER2 & & 5 & 6 & 21 & & 32 & 0 & \\
\hline Luminal & & 30 & 14 & 113 & & 152 & 5 & \\
\hline Triple-negative & 218 & & & & 0.006 & & & $<\mathbf{0 . 0 0 1}$ \\
\hline No & & 35 & 21 & 133 & & 183 & 6 & \\
\hline Yes & & 13 & 3 & 13 & & 20 & 9 & \\
\hline E-cadherin & 201 & & & & $<\mathbf{0 . 0 0 1}$ & & & 0.710 \\
\hline Negative & & 33 & 7 & 18 & & 53 & 5 & \\
\hline Reduced & & 3 & 2 & 9 & & 13 & 1 & \\
\hline Normal & & 9 & 13 & 107 & & 122 & 7 & \\
\hline TOP $2 A$ & 196 & & & & 0.365 & & & 0.266 \\
\hline Low & & 16 & 10 & 65 & & 87 & 4 & \\
\hline High & & 27 & 12 & 66 & & 95 & 10 & \\
\hline TOP2A CISH & 211 & & & & 0.201 & & & 0.610 \\
\hline Not amp & & 44 & 19 & 131 & & 180 & 14 & \\
\hline Amp & & 4 & 4 & 9 & & 17 & 0 & \\
\hline Cyclin D1 & 208 & & & & 0.044 & & & 0.057 \\
\hline Low & & 11 & 3 & 10 & & 20 & 4 & \\
\hline Intermediate & & 9 & 3 & 30 & & 38 & 4 & \\
\hline High & & 27 & 17 & 98 & & 136 & 6 & \\
\hline CCND1 CISH & 221 & & & & 0.185 & & & 1.000 \\
\hline Not amp & & 42 & 18 & 132 & & 179 & 13 & \\
\hline Amp & & 6 & 6 & 17 & & 27 & 2 & \\
\hline MYC CISH & 184 & & & & 0.725 & & & 0.021 \\
\hline Not amp & & 36 & 18 & 113 & & 158 & 9 & \\
\hline Amp & & 3 & 1 & 13 & & 13 & 4 & \\
\hline Caveolin 1 & 222 & & & & 0.040 & & & $<\mathbf{0 . 0 0 1}$ \\
\hline Negative & & 39 & 23 & 139 & & 193 & 8 & \\
\hline Positive & & 9 & 1 & 11 & & 14 & 7 & \\
\hline Caveolin 2 & 196 & & & & 0.484 & & & 0.001 \\
\hline Negative & & 41 & 20 & 123 & & 175 & 9 & \\
\hline Positive & & 3 & 0 & 9 & & 7 & 5 & \\
\hline Nestin & 167 & & & & 0.187 & & & 0.005 \\
\hline Negative & & 28 & 16 & 104 & & 141 & 7 & \\
\hline Positive & & 7 & 2 & 10 & & 14 & 5 & \\
\hline FOXA1 & 175 & & & & 0.046 & & & 0.001 \\
\hline Negative & & 16 & 4 & 23 & & 34 & 9 & \\
\hline Positive & & 25 & 13 & 94 & & 128 & 4 & \\
\hline Bcl2 & 171 & & & & 0.098 & & & 0.170 \\
\hline Negative & & 19 & 6 & 36 & & 55 & 6 & \\
\hline Positive & & 19 & 10 & 81 & & 106 & 4 & \\
\hline
\end{tabular}

amp: amplified; CISH: chromogenic in situ hybridization; ER: oestrogen receptor; IDC: invasive ductal carcinoma; LN mets: lymph node metastasis; LVI: lympho-vascular invasion; PR: progesterone receptor.

${ }^{\mathrm{a}}$ Molecular subtypes as defined by Nielsen immunohistochemical surrogate panel. ${ }^{34}$

Significant $P$-values are shown in bold.

$(P<0.05$, Tables 7 and 8$)$. Taken together, these results provide strong circumstantial evidence to suggest that Wnt pathway is preferentially activated in triple-negative/basal-like breast cancers.

Given the association between aberrant $\beta$-catenin expression and clinicopathological features asso- ciated with poor outcome, it is not surprising that univariate survival analysis revealed that $\beta$-catenin nuclear expression as defined by $14 / \beta$-catenin clone was significantly associated with decreased metastasis-free survival $(P=0.0216$, Figure 5$)$ and overall survival ( $P=0.0237$, Figure 5). Furthermore, a trend 
Table $5 \beta$-Catenin membranous and nuclear expression in 186 non-lobular invasive breast carcinomas (14/ $\beta$-catenin clone)

\begin{tabular}{|c|c|c|c|c|c|c|c|c|}
\hline Parameter & $\mathrm{N}$ & $\begin{array}{c}\text { Membranous } \\
\text { negative }\end{array}$ & $\begin{array}{l}\text { Membranous } \\
\text { reduced }\end{array}$ & $\begin{array}{c}\text { Membranous } \\
\text { normal }\end{array}$ & $\mathrm{P}$-value & $\begin{array}{l}\text { Nuclear } \\
\text { negative }\end{array}$ & $\begin{array}{l}\text { Nuclear } \\
\text { positive }\end{array}$ & $\mathrm{P}$-value \\
\hline Size-TNM & 186 & & & & 0.534 & & & 0.067 \\
\hline $\mathrm{T} 1$ & & 9 & 7 & 83 & & 92 & 7 & \\
\hline $\mathrm{T} 2$ & & 11 & 9 & 56 & & 62 & 14 & \\
\hline T3 & & 2 & 1 & 8 & & 10 & 1 & \\
\hline Grade & 183 & & & & 0.398 & & & 0.043 \\
\hline 1 & & 2 & 1 & 19 & & 22 & 0 & \\
\hline 2 & & 3 & 3 & 39 & & 42 & 3 & \\
\hline 3 & & 17 & 13 & 86 & & 97 & 19 & \\
\hline Tyре & 186 & & & & 0.342 & & & 0.148 \\
\hline IDC & & 16 & 15 & 129 & & 140 & 20 & \\
\hline Mixed & & 4 & 2 & 12 & & 18 & 0 & \\
\hline Other & & 2 & 0 & 6 & & 6 & 2 & \\
\hline$L V I$ & 185 & & & & 0.433 & & & 0.322 \\
\hline Negative & & 8 & 7 & 41 & & 47 & 9 & \\
\hline Positive & & 14 & 10 & 105 & & 116 & 13 & \\
\hline$L N$ mets & 181 & & & & 0.304 & & & 0.017 \\
\hline Negative & & 11 & 6 & 47 & & 51 & 13 & \\
\hline Positive & & 11 & 11 & 95 & & 108 & 9 & \\
\hline$E R$ & 186 & & & & 0.013 & & & $<0.001$ \\
\hline Negative & & 8 & 6 & 22 & & 21 & 15 & \\
\hline Positive & & 14 & 11 & 125 & & 143 & 7 & \\
\hline$P R$ & 186 & & & & 0.018 & & & $<0.001$ \\
\hline Negative & & 11 & 6 & 33 & & 35 & 15 & \\
\hline Positive & & 11 & 11 & 114 & & 129 & 7 & \\
\hline HER2 & 186 & & & & 0.029 & & & 0.028 \\
\hline Negative & & 21 & 11 & 125 & & 135 & 22 & \\
\hline Positive & & 1 & 6 & 22 & & 29 & 0 & \\
\hline HER2 CISH & 176 & & & & 0.031 & & & 0.028 \\
\hline Not amp & & 21 & 11 & 116 & & 127 & 21 & \\
\hline Amp & & 1 & 6 & 21 & & 28 & 0 & \\
\hline EGFR & 186 & & & & 0.200 & & & 0.002 \\
\hline Negative & & 18 & 14 & 135 & & 152 & 15 & \\
\hline Positive & & 4 & 3 & 12 & & 12 & 7 & \\
\hline CK14 & 185 & & & & 0.019 & & & $<0.001$ \\
\hline Negative & & 18 & 13 & 137 & & 156 & 12 & \\
\hline Positive & & 4 & 4 & 9 & & 7 & 10 & \\
\hline CK5/6 & 179 & & & & 0.214 & & & $<0.001$ \\
\hline Negative & & 17 & 13 & 128 & & 148 & 10 & \\
\hline Positive & & 5 & 2 & 14 & & 10 & 11 & \\
\hline CK17 & 184 & & & & 0.007 & & & $<0.001$ \\
\hline Negative & & 16 & 12 & 132 & & 147 & 13 & \\
\hline Positive & & 6 & 5 & 13 & & 15 & 9 & \\
\hline Basal CKs & 185 & & & & 0.021 & & & $<0.001$ \\
\hline Negative & & 15 & 12 & 128 & & 145 & 10 & \\
\hline Positive & & 7 & 5 & 18 & & 18 & 12 & \\
\hline $\begin{array}{l}\text { Basal CKs or } \\
\text { EGFR }\end{array}$ & 185 & & & & 0.006 & & & $<0.001$ \\
\hline Negative & & 14 & 11 & 126 & & 144 & 7 & \\
\hline Positive & & 8 & 6 & 20 & & 19 & 15 & \\
\hline P53 & 172 & & & & 0.005 & & & 0.005 \\
\hline Negative & & 10 & 8 & 103 & & 112 & 9 & \\
\hline Positive & & 11 & 8 & 32 & & 39 & 12 & \\
\hline$M I B-1$ & 171 & & & & 0.221 & & & $<0.001$ \\
\hline$<10 \%$ & & 5 & 4 & 57 & & 61 & 5 & \\
\hline $10-30 \%$ & & 10 & 9 & 59 & & 71 & 7 & \\
\hline$>30 \%$ & & 6 & 3 & 18 & & 17 & 10 & \\
\hline
\end{tabular}


Table 5 Continued

\begin{tabular}{|c|c|c|c|c|c|c|c|c|}
\hline Parameter & $\mathrm{N}$ & $\begin{array}{c}\text { Membranous } \\
\text { negative }\end{array}$ & $\begin{array}{l}\text { Membranous } \\
\text { reduced }\end{array}$ & $\begin{array}{c}\text { Membranous } \\
\text { normal }\end{array}$ & P-value & $\begin{array}{l}\text { Nuclear } \\
\text { negative }\end{array}$ & $\begin{array}{l}\text { Nuclear } \\
\text { positive }\end{array}$ & $\mathrm{P}$-value \\
\hline $\begin{array}{l}\text { Molecular } \\
\text { subtype }^{\text {a }}\end{array}$ & 182 & & & & $<0.001$ & & & $<\mathbf{0 . 0 0 1}$ \\
\hline Basal & & 7 & 5 & 14 & & 13 & 13 & \\
\hline HER2 & & 1 & 6 & 23 & & 30 & 0 & \\
\hline Luminal & & 13 & 5 & 108 & & 119 & 7 & \\
\hline Triple-negative & 182 & & & & 0.001 & & & $<0.001$ \\
\hline No & & 14 & 12 & 130 & & 148 & 8 & \\
\hline Yes & & 8 & 5 & 13 & & 12 & 14 & \\
\hline E-cadherin & 166 & & & & 0.016 & & & 0.047 \\
\hline Negative & & 8 & 4 & 16 & & 21 & 7 & \\
\hline Reduced & & 1 & 2 & 10 & & 12 & 1 & \\
\hline Normal & & 10 & 10 & 105 & & 114 & 11 & \\
\hline TOP $2 A$ & 163 & & & & 0.566 & & & 0.473 \\
\hline Low & & 7 & 6 & 60 & & 66 & 7 & \\
\hline High & & 13 & 9 & 68 & & 77 & 13 & \\
\hline TOP2A CISH & 175 & & & & 0.009 & & & 0.223 \\
\hline Not amp & & 20 & 12 & 127 & & 138 & 21 & \\
\hline Amp & & 1 & 5 & 10 & & 16 & 0 & \\
\hline Cyclin D1 & 173 & & & & 0.001 & & & $<\mathbf{0 . 0 0 1}$ \\
\hline Low & & 8 & 2 & 9 & & 12 & 7 & \\
\hline Intermediate & & 3 & 3 & 29 & & 28 & 7 & \\
\hline High & & 10 & 12 & 97 & & 112 & 7 & \\
\hline CCND1 CISH & 186 & & & & 0.757 & & & 0.320 \\
\hline Not amp & & 19 & 14 & 130 & & 142 & 21 & \\
\hline Amp & & 3 & 3 & 17 & & 22 & 1 & \\
\hline MYC CISH & 156 & & & & 0.710 & & & 1,000 \\
\hline Not amp & & 17 & 12 & 111 & & 123 & 17 & \\
\hline Amp & & 1 & 2 & 13 & & 14 & 2 & \\
\hline Caveolin 1 & 186 & & & & 0.016 & & & $<0.001$ \\
\hline Negative & & 16 & 15 & 136 & & 154 & 13 & \\
\hline Positive & & 6 & 2 & 11 & & 10 & 9 & \\
\hline Caveolin 2 & 164 & & & & 0.679 & & & $<0.001$ \\
\hline Negative & & 18 & 14 & 120 & & 139 & 13 & \\
\hline Positive & & 1 & 2 & 9 & & 5 & 7 & \\
\hline Nestin & 141 & & & & 0.026 & & & $<\mathbf{0 . 0 0 1}$ \\
\hline Negative & & 12 & 9 & 102 & & 114 & 9 & \\
\hline Positive & & 5 & 3 & 10 & & 9 & 9 & \\
\hline FOXA1 & 144 & & & & 0.001 & & & $<\mathbf{0 . 0 0 1}$ \\
\hline Negative & & 10 & 6 & 22 & & 27 & 11 & \\
\hline Positive & & 8 & 7 & 91 & & 100 & 6 & \\
\hline Bcl2 & 142 & & & & 0.048 & & & 0.087 \\
\hline Negative & & 9 & 8 & 36 & & 44 & 9 & \\
\hline Positive & & 7 & 6 & 76 & & 83 & 6 & \\
\hline
\end{tabular}

amp: amplified; CISH: chromogenic in situ hybridization; ER: oestrogen receptor; IDC: invasive ductal carcinoma; LN mets: lymph node metastasis; LVI: lympho-vascular invasion; PR: progesterone receptor.

${ }^{\mathrm{a}}$ Molecular subtypes as defined by Nielsen immunohistochemical surrogate panel. ${ }^{34}$

Significant $P$-values are shown in bold.

for an association between $\beta$-catenin nuclear expression and decreased disease-free survival was observed ( $P=0.0873$, Figure 5$)$. We did not observe any significant correlation between $\beta$-catenin membranous or cytoplasmic expression and survival (data not shown). Multivariate survival analysis demonstrated that $\beta$-catenin nuclear expression was not associated with the outcome of breast cancer patients when other clinicopathological parameters were included in the model (ie, tumour size, lymph node 
Table $6 \beta$-Catenin membranous and nuclear expression in 187 non-lobular invasive breast carcinomas (17C2 clone)

\begin{tabular}{|c|c|c|c|c|c|c|c|c|}
\hline Parameter & $\mathrm{N}$ & $\begin{array}{c}\text { Membranous } \\
\text { negative }\end{array}$ & $\begin{array}{l}\text { Membranous } \\
\text { reduced }\end{array}$ & $\begin{array}{c}\text { Membranous } \\
\text { positive }\end{array}$ & $\mathrm{P}$-value & $\begin{array}{l}\text { Nuclear } \\
\text { negative }\end{array}$ & $\begin{array}{l}\text { Nuclear } \\
\text { positive }\end{array}$ & P-value \\
\hline Size-TNM & 187 & & & & 0.314 & & & 0.954 \\
\hline $\mathrm{T} 1$ & & 10 & 7 & 83 & & 93 & 7 & \\
\hline $\mathrm{T} 2$ & & 10 & 12 & 54 & & 70 & 6 & \\
\hline T3 & & 2 & 1 & 8 & & 10 & 1 & \\
\hline Grade & 184 & & & & 0.353 & & & 0.291 \\
\hline 1 & & 1 & 2 & 19 & & 22 & 0 & \\
\hline 2 & & 3 & 4 & 39 & & 43 & 3 & \\
\hline 3 & & 18 & 13 & 85 & & 105 & 11 & \\
\hline Tyрe & 187 & & & & 0.473 & & & 0.082 \\
\hline IDC & & 17 & 17 & 127 & & 149 & 12 & \\
\hline Mixed & & 3 & 3 & 12 & & 18 & 0 & \\
\hline Other & & 2 & 0 & 6 & & 6 & 2 & \\
\hline$L V I$ & 186 & & & & 0.191 & & & 0.363 \\
\hline Negative & & 10 & 7 & 39 & & 50 & 6 & \\
\hline Positive & & 12 & 13 & 105 & & 122 & 8 & \\
\hline$L N$ mets & 182 & & & & 0.450 & & & 0.007 \\
\hline Negative & & 10 & 8 & 47 & & 55 & 10 & \\
\hline Positive & & 12 & 11 & 94 & & 113 & 4 & \\
\hline$E R$ & 187 & & & & 0.001 & & & $<0.001$ \\
\hline Negative & & 11 & 3 & 22 & & 26 & 10 & \\
\hline Positive & & 11 & 17 & 123 & & 147 & 4 & \\
\hline$P R$ & 187 & & & & 0.001 & & & $<0.001$ \\
\hline Negative & & 13 & 4 & 33 & & 40 & 10 & \\
\hline Positive & & 9 & 16 & 112 & & 133 & 4 & \\
\hline HER2 & 187 & & & & 0.461 & & & 0.132 \\
\hline Negative & & 19 & 15 & 124 & & 144 & 14 & \\
\hline Positive & & 3 & 5 & 21 & & 29 & 0 & \\
\hline HER2 CISH & 177 & & & & 0.182 & & & 0.227 \\
\hline Not amp & & 19 & 14 & 116 & & 136 & 13 & \\
\hline Amp & & 3 & 6 & 19 & & 28 & 0 & \\
\hline$E G F R$ & 187 & & & & 0.269 & & & 0.040 \\
\hline Negative & & 18 & 17 & 133 & & 158 & 10 & \\
\hline Positive & & 4 & 3 & 12 & & 15 & 4 & \\
\hline CK14 & 186 & & & & 0.028 & & & $<\mathbf{0 . 0 0 1}$ \\
\hline Negative & & 17 & 17 & 135 & & 161 & 8 & \\
\hline Positive & & 5 & 3 & 9 & & 11 & 6 & \\
\hline$C K 5 / 6$ & 180 & & & & 0.379 & & & 0.002 \\
\hline Negative & & 16 & 17 & 126 & & 151 & 8 & \\
\hline Positive & & 4 & 3 & 14 & & 15 & 6 & \\
\hline CK17 & 185 & & & & 0.015 & & & 0.089 \\
\hline Negative & & 16 & 15 & 130 & & 151 & 10 & \\
\hline Positive & & 6 & 5 & 13 & & 20 & 4 & \\
\hline Basal CKs & 186 & & & & 0.037 & & & 0.002 \\
\hline Negative & & 15 & 15 & 126 & & 149 & 7 & \\
\hline Positive & & 7 & 5 & 18 & & 23 & 7 & \\
\hline Basal CKs or EGFR & 186 & & & & 0.007 & & & $<\mathbf{0 . 0 0 1}$ \\
\hline Negative & & 13 & 15 & 124 & & 147 & 5 & \\
\hline Positive & & 9 & 5 & 20 & & 25 & 9 & \\
\hline P53 & 173 & & & & 0.004 & & & 0.059 \\
\hline Negative & & 9 & 12 & 101 & & 116 & 6 & \\
\hline Positive & & 13 & 6 & 32 & & 44 & 7 & \\
\hline MIB-1 & 172 & & & & 0.026 & & & 0.014 \\
\hline$<10 \%$ & & 2 & 9 & 56 & & 63 & 4 & \\
\hline $10-30 \%$ & & 12 & 8 & 58 & & 74 & 4 & \\
\hline$>30 \%$ & & 7 & 2 & 18 & & 21 & 6 & \\
\hline
\end{tabular}


Table 6 Continued

\begin{tabular}{|c|c|c|c|c|c|c|c|c|}
\hline Parameter & $\mathrm{N}$ & $\begin{array}{c}\text { Membranous } \\
\text { negative }\end{array}$ & $\begin{array}{l}\text { Membranous } \\
\text { reduced }\end{array}$ & $\begin{array}{c}\text { Membranous } \\
\text { positive }\end{array}$ & $\mathrm{P}$-value & $\begin{array}{l}\text { Nuclear } \\
\text { negative }\end{array}$ & $\begin{array}{l}\text { Nuclear } \\
\text { positive }\end{array}$ & $\mathrm{P}$-value \\
\hline Molecular subtype ${ }^{\mathrm{a}}$ & 183 & & & & $<\mathbf{0 . 0 0 1}$ & & & $<\mathbf{0 . 0 0 1}$ \\
\hline Basal & & 9 & 3 & 14 & & 18 & 8 & \\
\hline HER2 & & 3 & 6 & 21 & & 30 & 0 & \\
\hline Luminal & & 8 & 11 & 108 & & 123 & 4 & \\
\hline Triple-negative & 183 & & & & $<\mathbf{0 . 0 0 1}$ & & & $<\mathbf{0 . 0 0 1}$ \\
\hline No & & 11 & 18 & 128 & & 152 & 5 & \\
\hline Yes & & 11 & 2 & 13 & & 17 & 9 & \\
\hline E-cadherin & 167 & & & & 0.004 & & & 0.269 \\
\hline Negative & & 9 & 4 & 15 & & 24 & 4 & \\
\hline Reduced & & 2 & 2 & 9 & & 12 & 1 & \\
\hline Normal & & 9 & 12 & 105 & & 119 & 7 & \\
\hline TOP $2 A$ & 164 & & & & 0.490 & & & 0.146 \\
\hline Low & & 7 & 7 & 60 & & 71 & 3 & \\
\hline High & & 13 & 11 & 66 & & 80 & 10 & \\
\hline TOP2A CISH & 176 & & & & 0.091 & & & 0.611 \\
\hline Not amp & & 19 & 15 & 126 & & 147 & 13 & \\
\hline Amp & & 3 & 4 & 9 & & 16 & 0 & \\
\hline Cyclin D1 & 174 & & & & 0.002 & & & 0.021 \\
\hline Low & & 8 & 2 & 9 & & 15 & 4 & \\
\hline Intermediate & & 3 & 3 & 29 & & 31 & 4 & \\
\hline High & & 11 & 14 & 95 & & 115 & 5 & \\
\hline CCND1 CISH & 187 & & & & 0.181 & & & 1.000 \\
\hline Not amp & & 20 & 15 & 129 & & 151 & 13 & \\
\hline Amp & & 2 & 5 & 16 & & 22 & 1 & \\
\hline MYC CISH & 157 & & & & 0.860 & & & 0.108 \\
\hline Not amp & & 17 & 15 & 109 & & 132 & 9 & \\
\hline Amp & & 2 & 1 & 13 & & 13 & 3 & \\
\hline Caveolin 1 & 187 & & & & 0.002 & & & $<0.001$ \\
\hline Negative & & 15 & 19 & 134 & & 161 & 7 & \\
\hline Positive & & 7 & 1 & 11 & & 12 & 7 & \\
\hline Caveolin 2 & 165 & & & & 0.238 & & & 0.001 \\
\hline Negative & & 18 & 17 & 118 & & 145 & 8 & \\
\hline Positive & & 3 & 0 & 9 & & 7 & 5 & \\
\hline Nestin & 142 & & & & 0.001 & & & 0.005 \\
\hline Negative & & 10 & 14 & 100 & & 118 & 6 & \\
\hline Positive & & 7 & 1 & 10 & & 13 & 5 & \\
\hline FOXA1 & 145 & & & & $<\mathbf{0 . 0 0 1}$ & & & 0.003 \\
\hline Negative & & 12 & 4 & 22 & & 30 & 8 & \\
\hline Positive & & 6 & 10 & 91 & & 103 & 4 & \\
\hline Bcl2 & 143 & & & & 0.003 & & & 0.292 \\
\hline Negative & & 12 & 6 & 35 & & 48 & 5 & \\
\hline Positive & & 4 & 9 & 77 & & 86 & 4 & \\
\hline
\end{tabular}

amp: amplified; CISH: chromogenic in situ hybridization; ER: oestrogen receptor; IDC: invasive ductal carcinoma; LN mets: lymph node metastasis; LVI: lympho-vascular invasion; PR: progesterone receptor.

${ }^{\mathrm{a}}$ Molecular subtypes as defined by Nielsen immunohistochemical surrogate panel. ${ }^{34}$

Significant $P$-values are shown in bold.

metastasis, oestrogen receptor, progesterone receptor and HER2 status, proliferation index as assessed by MIB-1 immunostaining, and basal-like markers; data not shown). No significant correlation between $\beta$-catenin expression and outcome could be observed when using 17C2 clone (data not shown).

\section{Absence of CTNNB1 Mutation in Breast Carcinomas Displaying Aberrant $\beta$-Catenin Expression}

Given that aberrant $\beta$-catenin nuclear expression was found in $11 \%$ of the breast cancers studied, we investigated the presence of CTNNB1 mutations in 
a

$\beta$-catenin membranous expression $14 / \beta$-catenin clone $\mathrm{p}<0.001^{*}$

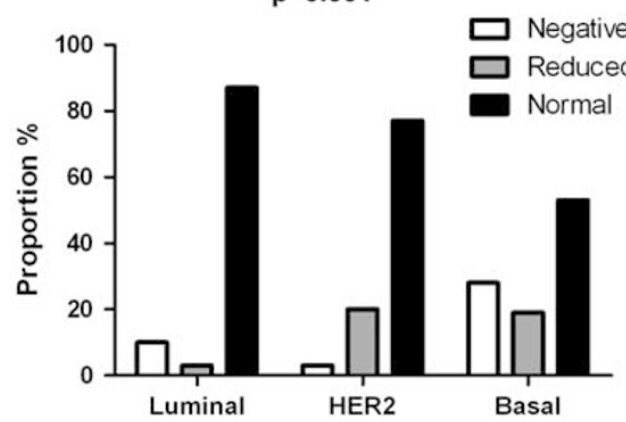

c

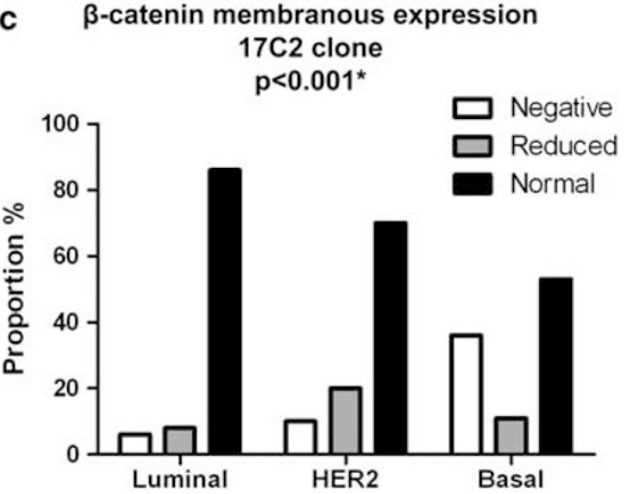

b $\beta$-catenin nuclear expression $14 / \beta$-catenin clone $\mathrm{p}<0.001^{*}$

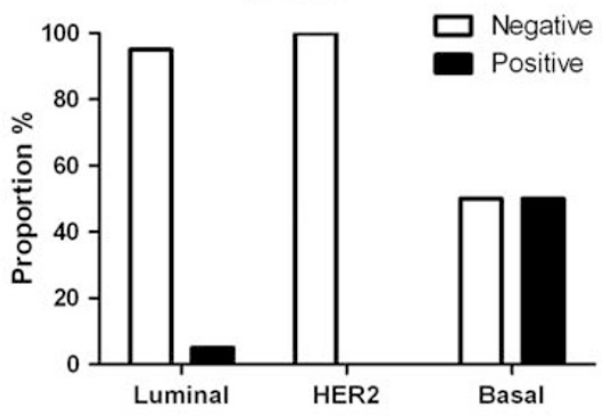

d

$\beta$-catenin nuclear expression 17C2 clone $\mathrm{p}<0.001^{\star}$

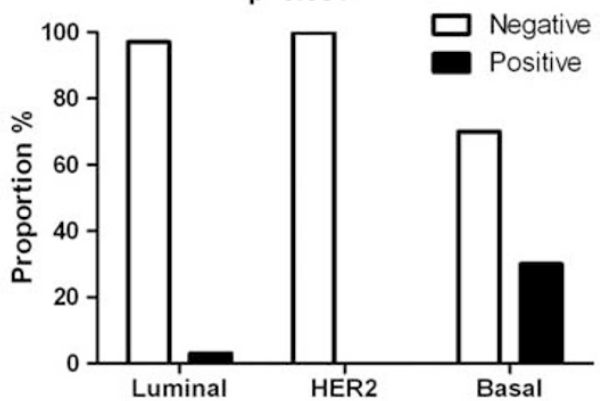

Figure $3 \beta$-Catenin expression in invasive breast carcinomas according to the molecular subtypes. $\beta$-Catenin membranous and nuclear expression using 14/ $\beta$-catenin (a, b) or 17C2 (c, d) clones in the cohort after exclusion of lobular carcinomas $(n=182 / 183$, respectively) according to the molecular subtypes of breast cancer as defined by the immunohistochemical surrogate described by Nielsen et al. ${ }^{34}$ ${ }^{*} \chi^{2}$ test.

28 cases, 19 with $\beta$-catenin nuclear accumulation and nine without, all of triple-negative phenotype. No exon 3 CTNNB1 gene mutations were found. Positive controls, included in each experiment, showed the expected presence of the previously reported CTNNB1 mutation on exon 3 (deletion of codon 45) in the HCT116 colorectal cancer cell line. $^{36}$ In addition, one formalin-fixed paraffinembedded sample of breast fibromatosis previously described $^{18}$ and displaying a CTNNB1 mutation on exon 3 (25195T > TC:45S > S/P) was also used as a positive control, confirming the accuracy of our sequencing technique. Taken together with our previous observations derived from the CTNNB1 sequencing of 21 metaplastic breast carcinomas, ${ }^{18}$ these results suggest that $\beta$-catenin/Wnt pathway activation in breast cancer is not commonly driven by CTNNB1 mutations.

\section{Discussion}

In this study we report that $\beta$-catenin expression in a large series of invasive breast carcinomas is aberrant in invasive lobular carcinomas and in a subgroup of triple-negative and basal-like breast cancers, as defined by a validated immunohistochemical surrogate panel. $^{34}$ Our results expand on those of
Khramtsov et al, ${ }^{19}$ as we have not only confirmed the association between $\beta$-catenin nuclear expression in basal-like cancers, but also demonstrated its association with proliferation markers, p53, CAV1, CAV2 and nestin..$^{34,37-39}$ We have also observed a statistically significant inverse correlation between $\beta$-catenin nuclear expression and lymph node metastasis at presentation, in the whole cohort $(P=0.044)$ and in the non-lobular $(P=0.017)$ and non-lobular grade $3(P=0.009)$ subgroups. This should not come as a surprise, given the reported lower frequency of lymph node metastasis in triplenegative $^{30}$ and basal-like ${ }^{41}$ cancers. Finally, our results further support our recent observations that metaplastic breast carcinomas, ${ }^{7,18}$ tumours that consistently display a basal-like phenotype, ${ }^{42-44}$ often display $\beta$-catenin aberrant expression but lack CTNNB1 gene mutations.

The prevalence of $\beta$-catenin/Wnt pathway activation and its association with outcome in breast cancer are contentious issues. Several studies have failed to demonstrate any association between $\beta$-catenin aberrant expression and outcome of breast cancer patients. ${ }^{11,12,14,16,20,45}$ Our data derived from the analysis of a cohort of 245 breast cancer patients uniformly treated with anthracycline-based chemotherapy and those from recent studies ${ }^{3,13,15,19}$ provide strong circumstantial evidence that aberrant 

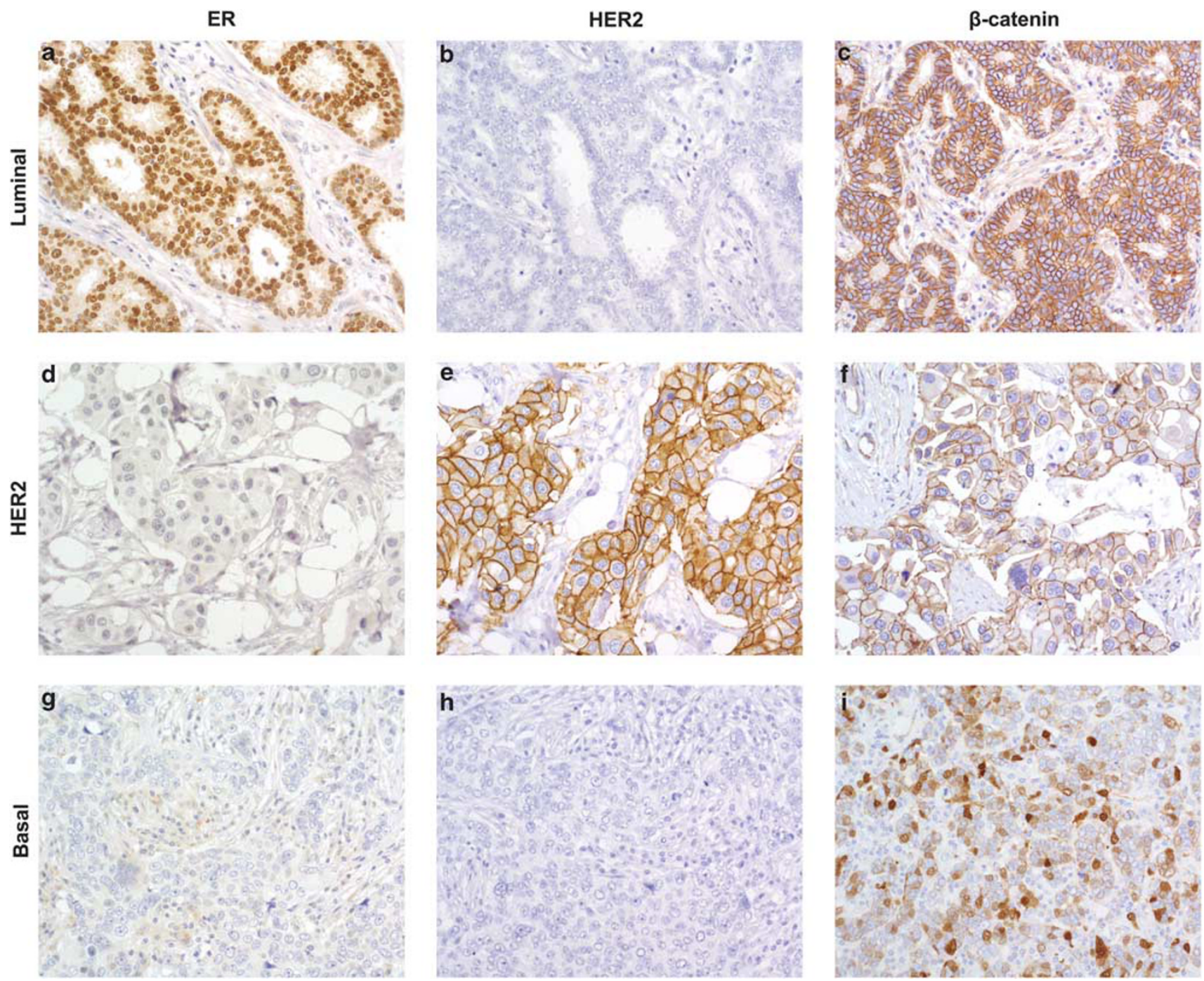

Figure $4 \beta$-Catenin expression in invasive breast carcinomas according to the molecular subtypes. One representative case of each subtype is shown: a luminal (ie, oestrogen receptor-positive (a) and HER2-negative (b)) carcinoma displaying $\beta$-catenin membranous expression without nuclear expression (c); a HER2 (ie, oestrogen receptor-negative (d) and HER2-positive (e)) carcinoma displaying $\beta$-catenin membranous expression without nuclear expression (f); and a basal-like (ie, oestrogen receptor-negative (g) and HER2-negative (h)) carcinoma showing strong $\beta$-catenin nuclear accumulation and lack of $\beta$-catenin membranous expression (i).

$\beta$-catenin expression is associated with a subset of patients of adverse outcome. It should be noted, however, that although $\beta$-catenin nuclear expression is significantly correlated with shorter metastasisfree and overall survival, it is not an independent predictor of outcome.

Although current evidence supports the contention that the $\beta$-catenin/Wnt pathway is activated in a subgroup of breast cancers, the mechanisms leading to $\beta$-catenin nuclear accumulation in breast cancer remain elusive. In this study we investigated the hypothesis that $\beta$-catenin nuclear expression would be driven by $C T N N B 1$ activating gene mutations as previously suggested by Hayes et al. ${ }^{25}$ No CTNNB1 mutations on exon 3 in 28 invasive breast carcinomas displaying $\beta$-catenin nuclear expression and/or triple-negative phenotype were found. This observation, which is in agreement with our previous
CTNNB1 mutation analysis in metaplastic breast carcinoma and studies from other groups, ${ }^{7,18}$ supports the hypothesis that $\beta$-catenin nuclear accumulation in breast cancer is not driven by mutations in CTNNB1 exon 3. Alternatively, $\beta$-catenin nuclear expression may stem from mutations affecting other exons of the CTNNB1 gene or other genes in the Wnt pathway (eg, the $A P C$ gene). It has been demonstrated that in colorectal cancers, copy number losses of $A P C$ gene may cooperate with inactivating $A P C$ mutations for complete APC protein loss of function and promote nuclear $\beta$-catenin translocation in tumour cells. ${ }^{46,47}$ Importantly, there is evidence to suggest that in breast cancer, APC mRNA expression levels are determined by $A P C$ gene copy numbers. ${ }^{47} \mathrm{We}$, therefore, performed a hypothesis generating re-analysis of microarraybased comparative genomic hybridization and gene 
Table $7 \beta$-Catenin membranous and nuclear expression in 116 grade 3 non-lobular invasive breast carcinomas (14/ $\beta$-catenin clone)

\begin{tabular}{|c|c|c|c|c|c|c|c|c|}
\hline Parameter & $\mathrm{N}$ & $\begin{array}{c}\text { Membranous } \\
\text { negative }\end{array}$ & $\begin{array}{l}\text { Membranous } \\
\text { reduced }\end{array}$ & $\begin{array}{c}\text { Membranous } \\
\text { normal }\end{array}$ & $\mathrm{P}$-value & $\begin{array}{l}\text { Nuclear } \\
\text { negative }\end{array}$ & $\begin{array}{l}\text { Nuclear } \\
\text { positive }\end{array}$ & $\mathrm{P}$-value \\
\hline Size-TNM & 116 & & & & 0.504 & & & 0.071 \\
\hline $\mathrm{T} 1$ & & 7 & 5 & 50 & & 56 & 6 & \\
\hline $\mathrm{T} 2$ & & 9 & 7 & 30 & & 34 & 12 & \\
\hline $\mathrm{T} 3$ & & 1 & 1 & 6 & & 7 & 1 & \\
\hline Grade & 116 & & & & NP & & & NP \\
\hline 3 & & 17 & 13 & 86 & & 97 & 19 & \\
\hline Type & 116 & & & & 0.211 & & & 0.265 \\
\hline IDC & & 14 & 12 & 82 & & 91 & 17 & \\
\hline Mixed & & 1 & 1 & 1 & & 3 & 0 & \\
\hline Other & & 2 & 0 & 3 & & 3 & 2 & \\
\hline$L V I$ & 116 & & & & 0.382 & & & 0.284 \\
\hline Negative & & 6 & 6 & 24 & & 28 & 8 & \\
\hline Positive & & 11 & 7 & 62 & & 69 & 11 & \\
\hline$L N$ mets & 113 & & & & 0.804 & & & 0.009 \\
\hline Negative & & 8 & 5 & 32 & & 32 & 13 & \\
\hline Positive & & 9 & 8 & 51 & & 62 & 6 & \\
\hline$E R$ & 116 & & & & 0.073 & & & $<0.001$ \\
\hline Negative & & 8 & 6 & 21 & & 20 & 15 & \\
\hline Positive & & 9 & 7 & 65 & & 77 & 4 & \\
\hline$P R$ & 116 & & & & 0.039 & & & 0.000 \\
\hline Negative & & 11 & 6 & 28 & & 30 & 15 & \\
\hline Positive & & 6 & 7 & 58 & & 67 & 4 & \\
\hline HER2 & 116 & & & & 0.195 & & & 0.012 \\
\hline Negative & & 16 & 9 & 66 & & 72 & 19 & \\
\hline Positive & & 1 & 4 & 20 & & 25 & 0 & \\
\hline HER2 CISH & 111 & & & & 0.209 & & & 0.021 \\
\hline Not amp & & 16 & 9 & 64 & & 71 & 18 & \\
\hline Amp & & 1 & 4 & 17 & & 22 & 0 & \\
\hline$E G F R$ & 116 & & & & 0.489 & & & 0.015 \\
\hline Negative & & 13 & 10 & 74 & & 85 & 12 & \\
\hline Positive & & 4 & 3 & 12 & & 12 & 7 & \\
\hline CK14 & 116 & & & & 0.083 & & & $<\mathbf{0 . 0 0 1}$ \\
\hline Negative & & 13 & 9 & 77 & & 90 & 9 & \\
\hline Positive & & 4 & 4 & 9 & & 7 & 10 & \\
\hline CK5/6 & 112 & & & & 0.392 & & & $<0.001$ \\
\hline Negative & & 12 & 9 & 71 & & 85 & 7 & \\
\hline Positive & & 5 & 2 & 13 & & 9 & 11 & \\
\hline CK17 & 116 & & & & 0.026 & & & 0.003 \\
\hline Negative & & 11 & 8 & 74 & & 83 & 10 & \\
\hline Positive & & 6 & 5 & 12 & & 14 & 9 & \\
\hline Basal CKs & 116 & & & & 0.087 & & & $<0.001$ \\
\hline Negative & & 10 & 8 & 69 & & 80 & 7 & \\
\hline Positive & & 7 & 5 & 17 & & 17 & 12 & \\
\hline Basal CKs or EGFR & 116 & & & & 0.037 & & & $<\mathbf{0 . 0 0 1}$ \\
\hline Negative & & 9 & 7 & 67 & & 79 & 4 & \\
\hline Positive & & 8 & 6 & 19 & & 18 & 15 & \\
\hline P53 & 109 & & & & 0.031 & & & 0.008 \\
\hline Negative & & 8 & 4 & 56 & & 62 & 6 & \\
\hline Positive & & 8 & 8 & 25 & & 29 & 12 & \\
\hline$M I B-1$ & 108 & & & & 0.722 & & & 0.009 \\
\hline$<10 \%$ & & 1 & 2 & 11 & & 12 & 2 & \\
\hline $10-30 \%$ & & 9 & 7 & 51 & & 60 & 7 & \\
\hline$>30 \%$ & & 6 & 3 & 18 & & 17 & 10 & \\
\hline
\end{tabular}


Table 7 Continued

\begin{tabular}{|c|c|c|c|c|c|c|c|c|}
\hline Parameter & $\mathrm{N}$ & $\begin{array}{l}\text { Membranous } \\
\text { negative }\end{array}$ & $\begin{array}{l}\text { Membranous } \\
\text { reduced }\end{array}$ & $\begin{array}{c}\text { Membranous } \\
\text { normal }\end{array}$ & P-value & $\begin{array}{l}\text { Nuclear } \\
\text { negative }\end{array}$ & $\begin{array}{l}\text { Nuclear } \\
\text { positive }\end{array}$ & $\mathrm{P}$-value \\
\hline Molecular subtype & 112 & & & & 0.023 & & & $<\mathbf{0 . 0 0 1}$ \\
\hline Basal & & 7 & 5 & 14 & & 13 & 13 & \\
\hline HER2 & & 1 & 4 & 19 & & 24 & 0 & \\
\hline Luminal & & 8 & 3 & 51 & & 58 & 4 & \\
\hline Triple-negative & 114 & & & & 0.007 & & & $<\mathbf{0 . 0 0 1}$ \\
\hline No & & 9 & 8 & 71 & & 83 & 5 & \\
\hline Yes & & 8 & 5 & 13 & & 12 & 14 & \\
\hline E-cadherin & 105 & & & & 0.027 & & & 0.015 \\
\hline Negative & & 7 & 2 & 10 & & 12 & 7 & \\
\hline Reduced & & 1 & 2 & 6 & & 8 & 1 & \\
\hline Normal & & 7 & 8 & 62 & & 69 & 8 & \\
\hline TOP $2 A$ & 104 & & & & 0.152 & & & 0.789 \\
\hline Low & & 4 & 3 & 35 & & 36 & 6 & \\
\hline High & & 12 & 9 & 41 & & 51 & 11 & \\
\hline TOP2A CISH & 108 & & & & 0.362 & & & 0.120 \\
\hline Not amp & & 15 & 10 & 70 & & 77 & 18 & \\
\hline Amp & & 1 & 3 & 9 & & 13 & 0 & \\
\hline Cyclin D1 & 110 & & & & 0.008 & & & $<\mathbf{0 . 0 0 1}$ \\
\hline Low & & 7 & 2 & 7 & & 9 & 7 & \\
\hline Intermediate & & 3 & 2 & 15 & & 14 & 6 & \\
\hline High & & 6 & 9 & 59 & & 69 & 5 & \\
\hline CCND1 CISH & 116 & & & & 0.638 & & & 0.126 \\
\hline Not amp & & 16 & 11 & 74 & & 82 & 19 & \\
\hline Amp & & 1 & 2 & 12 & & 15 & 0 & \\
\hline MYC CISH & 99 & & & & 0.635 & & & 1.000 \\
\hline Not amp & & 13 & 8 & 66 & & 73 & 14 & \\
\hline Amp & & 1 & 2 & 9 & & 10 & 2 & \\
\hline Caveolin 1 & 116 & & & & 0.048 & & & $<0.001$ \\
\hline Negative & & 11 & 11 & 76 & & 88 & 10 & \\
\hline Positive & & 6 & 2 & 10 & & 9 & 9 & \\
\hline Caveolin 2 & 105 & & & & 0.672 & & & $<0.001$ \\
\hline Negative & & 15 & 10 & 69 & & 84 & 10 & \\
\hline Positive & & 1 & 2 & 8 & & 4 & 7 & \\
\hline Nestin & 89 & & & & 0.091 & & & $<\mathbf{0 . 0 0 1}$ \\
\hline Negative & & 8 & 6 & 57 & & 65 & 6 & \\
\hline Positive & & 5 & 3 & 10 & & 9 & 9 & \\
\hline FOXA1 & 90 & & & & 0.016 & & & $<0.001$ \\
\hline Negative & & 8 & 5 & 15 & & 17 & 11 & \\
\hline Positive & & 6 & 5 & 51 & & 58 & 4 & \\
\hline Bcl2 & 88 & & & & 0.424 & & & 0.245 \\
\hline Negative & & 7 & 7 & 28 & & 33 & 9 & \\
\hline Positive & & 6 & 4 & 36 & & 41 & 5 & \\
\hline
\end{tabular}

amp: amplified; CISH: chromogenic in situ hybridization; ER: oestrogen receptor; IDC: invasive ductal carcinoma; LN mets: lymph node metastasis; LVI: lympho-vascular invasion; PR: progesterone receptor.

${ }^{\mathrm{a}}$ Molecular subtypes as defined by Nielsen immunohistochemical surrogate panel. ${ }^{34}$

Significant $P$-values are shown in bold.

expression data from a previously published cohort of 95 grade 3 invasive breast cancers, ${ }^{48,49}$ which revealed a significant correlation between heterozygous deletions of $A P C$ locus and the basal-like phenotype ( $A P C$ loss in the basal-like subtype $n=10 / 25$, vs $2 / 25$ in the HER2 subtype and $4 / 45$ in the luminal subtype, $P=0.003,2 \times 3$ Fisher's exact test), and that in the basal-like tumours with $A P C$ loss, APC mRNA expression levels were significantly lower (Figures 6 and 7). Taken together, our results warrant further studies to determine whether $A P C$ deletions, mutations and gene promoter methy- 
Table $8 \beta$-Catenin membranous and nuclear expression in 116 grade 3 non-lobular invasive breast carcinomas (17C2 clone)

\begin{tabular}{|c|c|c|c|c|c|c|c|c|}
\hline Parameter & $\mathrm{N}$ & $\begin{array}{c}\text { Membranous } \\
\text { negative }\end{array}$ & $\begin{array}{l}\text { Membranous } \\
\text { reduced }\end{array}$ & $\begin{array}{c}\text { Membranous } \\
\text { positive }\end{array}$ & $\mathrm{P}$-value & $\begin{array}{l}\text { Nuclear } \\
\text { negative }\end{array}$ & $\begin{array}{l}\text { Nuclear } \\
\text { positive }\end{array}$ & $\mathrm{P}$-value \\
\hline Size-TNM & 116 & & & & 0.446 & & & 0.847 \\
\hline $\mathrm{T} 1$ & & 9 & 4 & 49 & & 57 & 5 & \\
\hline $\mathrm{T} 2$ & & 8 & 8 & 30 & & 41 & 5 & \\
\hline T3 & & 1 & 1 & 6 & & 7 & 1 & \\
\hline Tyре & 116 & & & & 0.056 & & & 0.052 \\
\hline IDC & & 14 & 13 & 81 & & 99 & 9 & \\
\hline Mixed & & 2 & 0 & 1 & & 3 & 0 & \\
\hline Other & & 2 & 0 & 3 & & 3 & 2 & \\
\hline$L V I$ & 116 & & & & 0.290 & & & 0.313 \\
\hline Negative & & 8 & 5 & 23 & & 31 & 5 & \\
\hline Positive & & 10 & 8 & 62 & & 74 & 6 & \\
\hline$L N$ mets & 113 & & & & 0.772 & & & 0.006 \\
\hline Negative & & 8 & 6 & 31 & & 36 & 9 & \\
\hline Positive & & 10 & 7 & 51 & & 66 & 2 & \\
\hline$E R$ & 116 & & & & 0.008 & & & $<\mathbf{0 . 0 0 1}$ \\
\hline Negative & 110 & 11 & 3 & 21 & & 25 & 10 & \\
\hline Positive & & 7 & 10 & 64 & & 80 & 1 & \\
\hline$P R$ & 116 & & & & 0.007 & & & $<0.001$ \\
\hline Negative & & 13 & 4 & 28 & & 35 & 10 & \\
\hline Positive & & 5 & 9 & 57 & & 70 & 1 & \\
\hline HER2 & 116 & & & & 0.503 & & & 0.118 \\
\hline Negative & & 16 & 10 & 65 & & 80 & 11 & \\
\hline Positive & & 2 & 3 & 20 & & 25 & 0 & \\
\hline HER2 CISH & 111 & & & & 0.398 & & & 0.206 \\
\hline Not amp & & 16 & 9 & 64 & & 79 & 10 & \\
\hline Amp & & 2 & 4 & 16 & & 22 & 0 & \\
\hline$E G F R$ & 116 & & & & 0.551 & & & 0.080 \\
\hline Negative & & 14 & 10 & 73 & & 90 & 7 & \\
\hline Positive & & 4 & 3 & 12 & & 15 & 4 & \\
\hline CK14 & 116 & & & & 0.114 & & & 0.001 \\
\hline Negative & & 13 & 10 & 76 & & 94 & 5 & \\
\hline Positive & & 5 & 3 & 9 & & 11 & 6 & \\
\hline CK5/6 & 112 & & & & 0.586 & & & 0.004 \\
\hline Negative & & 12 & 10 & 70 & & 87 & 5 & \\
\hline Positive & & 4 & 3 & 13 & & 14 & 6 & \\
\hline$C K 17$ & 116 & & & & 0.036 & & & 0.225 \\
\hline Negative & & 12 & 8 & 73 & & 86 & 7 & \\
\hline Positive & & 6 & 5 & 12 & & 19 & 4 & \\
\hline Basal CKs & 116 & & & & 0.120 & & & 0.005 \\
\hline Negative & & 11 & 8 & 68 & & 83 & 4 & \\
\hline Positive & & 7 & 5 & 17 & & 22 & 7 & \\
\hline Basal CKs or EGFR & 116 & & & & 0.043 & & & $<\mathbf{0 . 0 0 1}$ \\
\hline Negative & & 9 & 8 & 66 & & 81 & 2 & \\
\hline Positive & & 9 & 5 & 19 & & 24 & 9 & \\
\hline P53 & 109 & & & & 0.020 & & & 0.039 \\
\hline Negative & & 6 & 7 & 55 & & 65 & 3 & \\
\hline Positive & & 12 & 4 & 25 & & 34 & 7 & \\
\hline$M I B-1$ & 108 & & & & 0.203 & & & 0.057 \\
\hline$<10 \%$ & & 0 & 3 & 11 & & 13 & 1 & \\
\hline $10-30 \%$ & & 10 & 7 & 50 & & 63 & 4 & \\
\hline$>30 \%$ & & 7 & 2 & 18 & & 21 & 6 & \\
\hline
\end{tabular}


Table 8 Continued

\begin{tabular}{|c|c|c|c|c|c|c|c|c|}
\hline Parameter & $\mathrm{N}$ & $\begin{array}{c}\text { Membranous } \\
\text { negative }\end{array}$ & $\begin{array}{l}\text { Membranous } \\
\text { reduced }\end{array}$ & $\begin{array}{c}\text { Membranous } \\
\text { positive }\end{array}$ & $\mathrm{P}$-value & $\begin{array}{l}\text { Nuclear } \\
\text { negative }\end{array}$ & $\begin{array}{l}\text { Nuclear } \\
\text { positive }\end{array}$ & $\mathrm{P}$-value \\
\hline Molecular subtype & 112 & & & & 0.014 & & & $<\mathbf{0 . 0 0 1}$ \\
\hline Basal & & 9 & 3 & 14 & & 18 & 8 & \\
\hline HER2 & & 2 & 4 & 18 & & 24 & 0 & \\
\hline Luminal & & 5 & 6 & 51 & & 61 & 1 & \\
\hline Triple-negative & 114 & & & & $<\mathbf{0 . 0 0 1}$ & & & $<\mathbf{0 . 0 0 1}$ \\
\hline No & & 7 & 11 & 70 & & 86 & 2 & \\
\hline Yes & & 11 & 2 & 13 & & 17 & 9 & \\
\hline E-cadherin & 105 & & & & 0.005 & & & 0.083 \\
\hline Negative & & 8 & 2 & 9 & & 15 & 4 & \\
\hline Reduced & & 2 & 2 & 5 & & 8 & 1 & \\
\hline Normal & & 7 & 7 & 63 & & 73 & 4 & \\
\hline TOP $2 A$ & 104 & & & & 0.184 & & & 0.197 \\
\hline Low & & 6 & 2 & 34 & & 40 & 2 & \\
\hline High & & 12 & 9 & 41 & & 54 & 8 & \\
\hline TOP2A CISH & 108 & & & & 0.341 & & & 0.606 \\
\hline Not amp & & 16 & 9 & 70 & & 85 & 10 & \\
\hline Amp & & 2 & 3 & 8 & & 13 & 0 & \\
\hline Cyclin D1 & 110 & & & & 0.003 & & & 0.018 \\
\hline Low & & 8 & 1 & 7 & & 12 & 4 & \\
\hline Intermediate & & 3 & 2 & 15 & & 17 & 3 & \\
\hline High & & 7 & 9 & 58 & & 71 & 3 & \\
\hline CCND1 CISH & 116 & & & & 0.593 & & & 0.354 \\
\hline Not amp & & 17 & 11 & 73 & & 90 & 11 & \\
\hline Amp & & 1 & 2 & 12 & & 15 & 0 & \\
\hline MYC CISH & 99 & & & & 0.969 & & & 0.299 \\
\hline Not amp & & 13 & 9 & 65 & & 80 & 7 & \\
\hline Amp & & 2 & 1 & 9 & & 10 & 2 & \\
\hline Caveolin 1 & 116 & & & & 0.011 & & & $<0.001$ \\
\hline Negative & & 11 & 12 & 75 & & 94 & 4 & \\
\hline Positive & & 7 & 1 & 10 & & 11 & 7 & \\
\hline Caveolin 2 & 105 & & & & 0.364 & & & 0.001 \\
\hline Negative & & 15 & 11 & 68 & & 89 & 5 & \\
\hline Positive & & 3 & 0 & 8 & & 6 & 5 & \\
\hline Nestin & 89 & & & & 0.010 & & & 0.008 \\
\hline Negative & & 7 & 8 & 56 & & 68 & 3 & \\
\hline Positive & & 7 & 1 & 10 & & 13 & 5 & \\
\hline FOXA1 & 90 & & & & 0.001 & & & 0.001 \\
\hline Negative & & 11 & 2 & 15 & & 20 & 8 & \\
\hline Positive & & 4 & 7 & 51 & & 60 & 2 & \\
\hline Bcl2 & 88 & & & & 0.030 & & & 0.251 \\
\hline Negative & & 11 & 3 & 28 & & 37 & 5 & \\
\hline Positive & & 3 & 7 & 36 & & 44 & 2 & \\
\hline
\end{tabular}

amp: amplified; CISH: chromogenic in situ hybridization; ER: oestrogen receptor; IDC: invasive ductal carcinoma; LN mets: lymph node metastasis; LVI: lympho-vascular invasion; PR: progesterone receptor.

${ }^{\mathrm{a}}$ Molecular subtypes as defined by Nielsen immunohistochemical surrogate panel. ${ }^{34}$

Significant $P$-values are shown in bold.

lation may be the driver of $\beta$-catenin/Wnt pathway activation in a subgroup of basal-like and triplenegative breast cancers.

A significant correlation between $\beta$-catenin aberrant expression and reduction/lack of E-cadherin was found in the non-lobular and non-lobular grade
3 subgroups $(P<0.05$, Tables $5-8)$. We and others ${ }^{29,50}$ have previously shown that reduction of E-cadherin expression, one of the surrogate markers of epithelial-to-mesenchymal transition, ${ }^{50}$ is associated with non-lobular breast carcinomas of basal-like and triple-negative phenotypes. ${ }^{29}$ Hence, it is plausible 

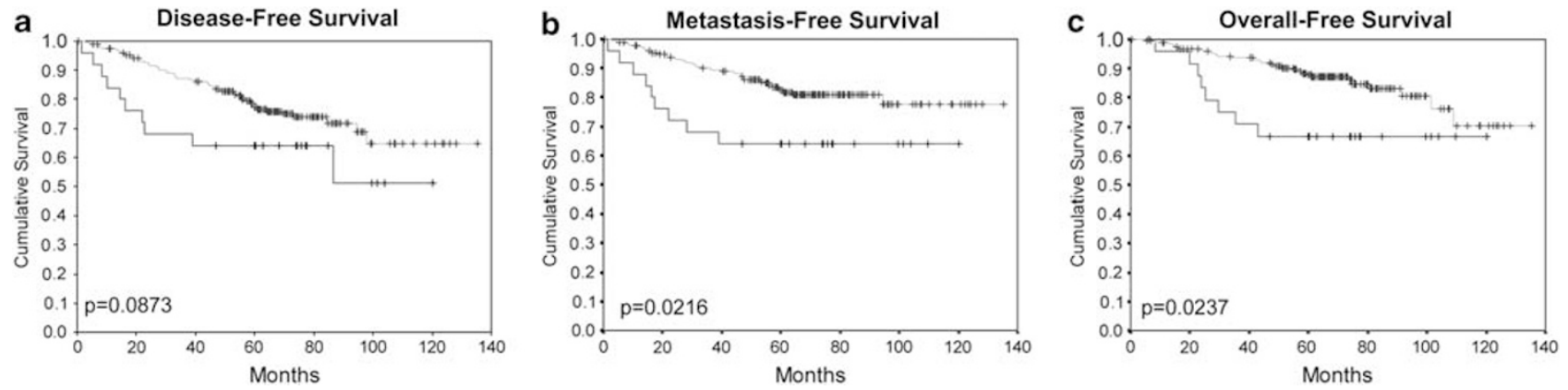

Figure $5 \beta$-Catenin nuclear expression is associated with poor survival. Kaplan-Meier curves for disease-free survival (a), metastasis-free survival (b) and overall survival (c) for 221 patients uniformly treated with anthracycline-based chemotherapy. Invasive breast carcinomas with $\beta$-catenin nuclear expression as defined by the $14 / \beta$-catenin clone (dark grey) are significantly associated with shorter metastasis-free survival and overall survival $(P<0.05)$. A similar but non-significant trend was also observed with disease-free survival.

a

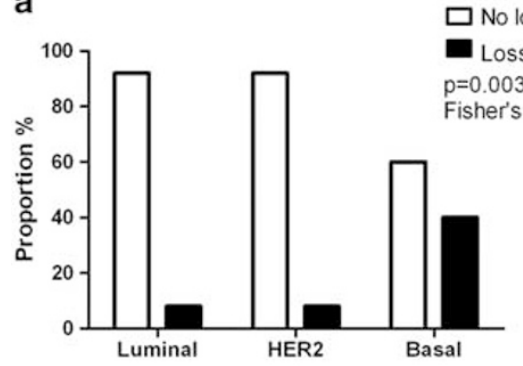

b

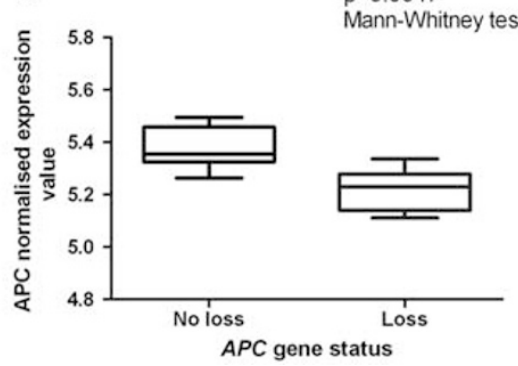

Figure $6 A P C$ (5q22.2) copy number and expression in the molecular subtypes of breast cancer. (a) Frequency of $A P C$ (5q22.2) loss, as defined by microarray-based comparative genomic hybridization performed with the 32K bacterial artificial chromosome tiling path array, ${ }^{48}$ in a previously published cohort of 95 grade 3 invasive breast cancers ${ }^{48,49}$ according to the molecular subtypes of breast cancer as defined by the immunohistochemical surrogate described by Nielsen et al. ${ }^{34}$ (b) Correlations between APC mRNA expression levels, as defined by the WG6 Illumina expression arrays (ArrayExpress http://www.ebi.ac.uk/microarray-as/ae/; accession number E-TABM$543)^{49}$ and $A P C$ loss (as defined by microarray-based comparative genomic hybridization performed with the $32 \mathrm{~K}$ bacterial artificial chromosome tiling path array $\left.{ }^{48}\right)$ in basal-like breast cancers $(n=13)$.

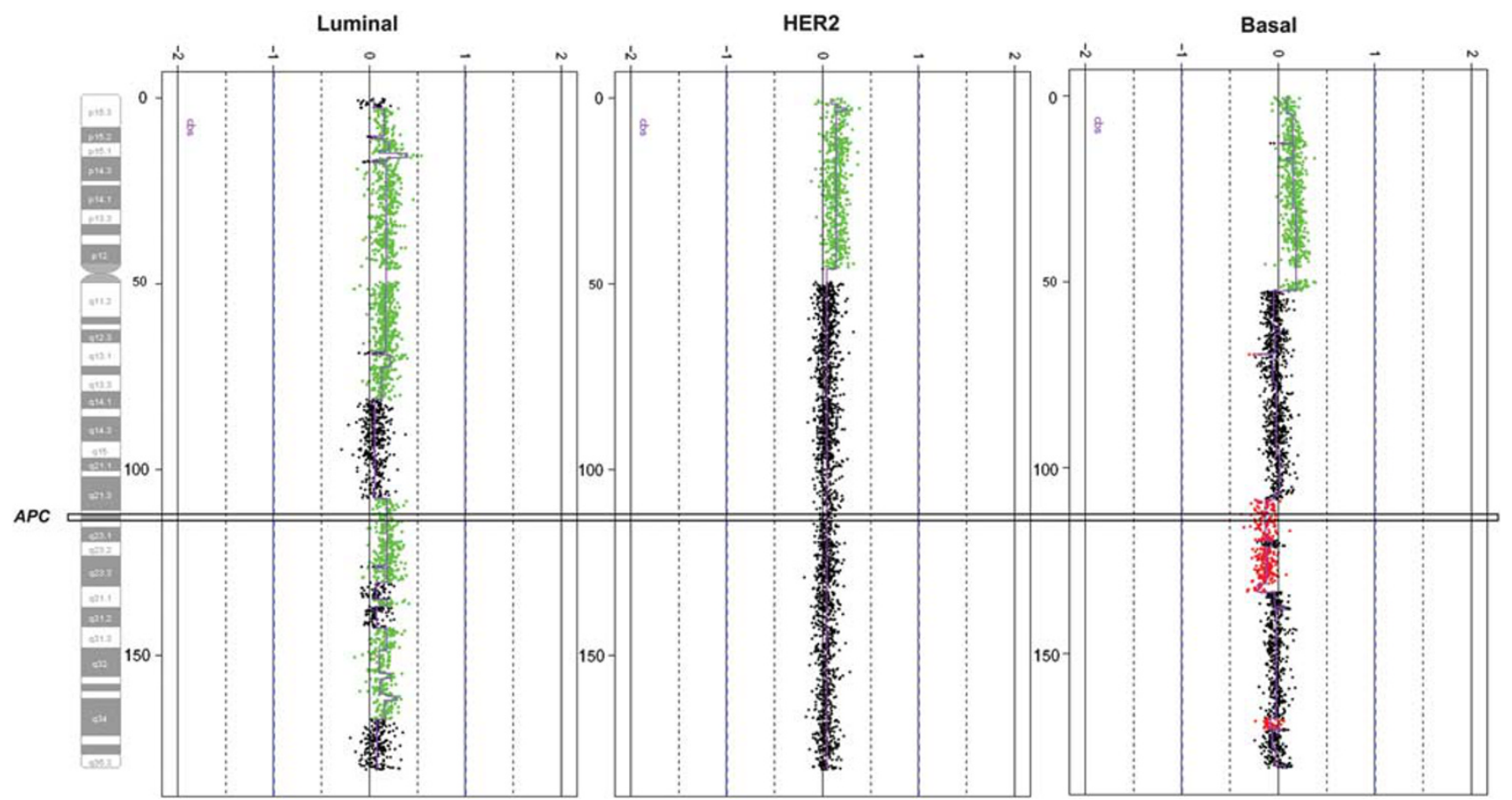

Figure 7 APC (5q22.2) copy number in the molecular subtypes of breast cancer. Representative chromosome 5 plots of luminal, HER2 and basal-like cancers obtained from re-analysis of the data from Natrajan et al. ${ }^{48} \log _{2}$ ratios are plotted on the $x$ axis against each clone according to genomic location on the $y$ axis. BACs categorized as displaying genomic gains or amplification are plotted in green and those categorized as genomic losses in red, as defined by previously validated cutoffs for circular binary segmentation-smoothed $\log _{2}$ ratios. 
that the activation of the $\beta$-catenin/Wnt pathway in these tumours may be the consequence of a global epithelial-to-mesenchymal transition programme, which is characteristic of at least a subset of basal-like tumours, including metaplastic breast cancers. ${ }^{50,51}$

In conclusion, $\beta$-catenin loss of membrane expression is a characteristic feature of invasive lobular carcinomas. $\beta$-catenin/Wnt canonical pathway activation, as defined by nuclear expression, is found in a subgroup of invasive breast cancers of triple-negative and basal-like phenotypes and is unlikely to be driven by CTNNB1 activating gene mutations. Studies exploring alternative mechanisms of $\beta$-catenin/Wnt pathway activation are warranted.

\section{Acknowledgements}

This study was funded in part by Breakthrough Breast Cancer. Magali Lacroix-Triki is funded by a partenariat grant from the Fédération Nationale des Centres de Lutte Contre le Cancer (FNCLCC, Paris) and the Fondation Médicale de France (Paris), and Monica Arnedos is funded by the Cridlan Fund.

\section{Disclosure/conflict of interest}

The authors declare no conflict of interest.

\section{References}

1 Hatsell S, Rowlands T, Hiremath M, et al. Beta-catenin and Tcfs in mammary development and cancer. J Mammary Gland Biol Neoplasia 2003;8:145-158.

$2 \mathrm{He}$ TC, Sparks AB, Rago C, et al. Identification of c-MYC as a target of the APC pathway. Science 1998;281:1509-1512.

3 Lin SY, Xia W, Wang JC, et al. Beta-catenin, a novel prognostic marker for breast cancer: its roles in cyclin D1 expression and cancer progression. Proc Natl Acad Sci USA 2000;97:4262-4266.

4 Tetsu O, McCormick F. Beta-catenin regulates expression of cyclin D1 in colon carcinoma cells. Nature 1999;398:422-426.

5 Cui J, Zhou X, Liu Y, et al. Wnt signaling in hepatocellular carcinoma: analysis of mutation and expression of beta-catenin, T-cell factor-4 and glycogen synthase kinase 3-beta genes. J Gastroenterol Hepatol 2003;18:280-287.

6 Deng J, Miller SA, Wang HY, et al. beta-catenin interacts with and inhibits NF-kappa B in human colon and breast cancer. Cancer Cell 2002;2:323-334.

7 Hennessy BT, Gonzalez-Angulo AM, Stemke-Hale K, et al. Characterization of a naturally occurring breast cancer subset enriched in epithelial-to-mesenchymal transition and stem cell characteristics. Cancer Res 2009;69:4116-4124.

8 Nagasawa Y, Miyoshi Y, Iwao K, et al. Transformation and morphological changes of murine L cells by transfection with a mutated form of beta-catenin. Cancer Res 1999;59:3539-3542.
9 Sparks AB, Morin PJ, Vogelstein B, et al. Mutational analysis of the APC/beta-catenin/Tcf pathway in colorectal cancer. Cancer Res 1998;58:1130-1134.

10 Bankfalvi A, Terpe HJ, Breukelmann D, et al. Immunophenotypic and prognostic analysis of E-cadherin and beta-catenin expression during breast carcinogenesis and tumour progression: a comparative study with CD44. Histopathology 1999;34:25-34.

11 Bukholm IK, Nesland JM, Karesen R, et al. E-cadherin and alpha-, beta-, and gamma-catenin protein expression in relation to metastasis in human breast carcinoma. J Pathol 1998;185:262-266.

12 Chung GG, Zerkowski MP, Ocal IT, et al. beta-Catenin and p53 analyses of a breast carcinoma tissue microarray. Cancer 2004;100:2084-2092.

13 Dolled-Filhart M, McCabe A, Giltnane J, et al. Quantitative in situ analysis of beta-catenin expression in breast cancer shows decreased expression is associated with poor outcome. Cancer Res 2006;66: 5487-5494.

14 Gillett CE, Miles DW, Ryder K, et al. Retention of the expression of E-cadherin and catenins is associated with shorter survival in grade III ductal carcinoma of the breast. J Pathol 2001;193:433-441.

15 Lopez-Knowles E, Zardawi SJ, McNeil CM, et al. Cytoplasmic localization of beta-catenin is a marker of poor outcome in breast cancer patients. Cancer Epidemiol Biomarkers Prev 2010;19:301-309.

16 Pedersen KB, Nesland JM, Fodstad O, et al. Expression of S100A4, E-cadherin, alpha- and beta-catenin in breast cancer biopsies. Br J Cancer 2002;87:1281-1286.

17 Wong SC, Lo SF, Lee KC, et al. Expression of frizzledrelated protein and Wnt-signalling molecules in invasive human breast tumours. J Pathol 2002; 196:145-153.

18 Lacroix-Triki M, Geyer FC, Lambros MB, et al. beta-catenin/Wnt signalling pathway in fibromatosis, metaplastic carcinomas and phyllodes tumours of the breast. Mod Pathol 2010; doi:10.1038/modpathol.2010.141 (e-pub ahead of print).

19 Khramtsov AI, Khramtsova GF, Tretiakova M, et al. Wnt/beta-catenin pathway activation is enriched in basal-like breast cancers and predicts poor outcome. Am J Pathol 2010;176:2911-2920.

20 Dillon DA, D’Aquila T, Reynolds AB, et al. The expression of p120ctn protein in breast cancer is independent of alpha- and beta-catenin and E-cadherin. Am J Pathol 1998;152:75-82.

21 Abraham SC, Reynolds C, Lee JH, et al. Fibromatosis of the breast and mutations involving the APC/ beta-catenin pathway. Hum Pathol 2002;33:39-46.

22 Candidus S, Bischoff P, Becker KF, et al. No evidence for mutations in the alpha- and beta-catenin genes in human gastric and breast carcinomas. Cancer Res 1996;56:49-52.

23 Lazar AJ, Tuvin D, Hajibashi S, et al. Specific mutations in the beta-catenin gene (CTNNB1) correlate with local recurrence in sporadic desmoid tumors. Am J Pathol 2008;173:1518-1527.

24 Miyaki M, Konishi M, Kikuchi-Yanoshita $\mathrm{R}$, et al. Coexistence of somatic and germ-line mutations of APC gene in desmoid tumors from patients with familial adenomatous polyposis. Cancer Res 1993;53:5079-5082.

25 Hayes MJ, Thomas D, Emmons A, et al. Genetic changes of Wnt pathway genes are common events in metaplastic carcinomas of the breast. Clin Cancer Res 2008;14:4038-4044. 
26 Elston CW, Ellis IO. Pathological prognostic factors in breast cancer I. The value of histological grade in breast cancer: experience from a large study with longterm follow-up. Histopathology 1991;19:403-410.

27 Singletary SE, Connolly JL. Breast cancer staging: working with the sixth edition of the AJCC Cancer Staging Manual. CA Cancer J Clin 2006;56:37-47, quiz 50-1.

28 Harvey JM, Clark GM, Osborne CK, et al. Estrogen receptor status by immunohistochemistry is superior to the ligand-binding assay for predicting response to adjuvant endocrine therapy in breast cancer. J Clin Oncol 1999;17:1474-1481.

29 Mahler-Araujo B, Savage K, Parry S, et al. Reduction of E-cadherin expression is associated with non-lobular breast carcinomas of basal-like and triple negative phenotype. J Clin Pathol 2008;61:615-620.

30 Tan DS, Marchio C, Jones RL, et al. Triple negative breast cancer: molecular profiling and prognostic impact in adjuvant anthracycline-treated patients. Breast Cancer Res Treat 2008;111:27-44.

31 Lambros MB, Natrajan R, Geyer FC, et al. PPM1D gene amplification and overexpression in breast cancer: a qRT-PCR and chromogenic in situ hybridization study. Mod Pathol 2010;23:1334.

32 Arriola E, Marchio C, Tan DS, et al. Genomic analysis of the HER2/TOP2A amplicon in breast cancer and breast cancer cell lines. Lab Invest 2008;88:491-503.

33 Reis-Filho JS, Savage K, Lambros MB, et al. Cyclin D1 protein overexpression and CCND1 amplification in breast carcinomas: an immunohistochemical and chromogenic in situ hybridisation analysis. Mod Pathol 2006;19:999-1009.

34 Nielsen TO, Hsu FD, Jensen $\mathrm{K}$, et al. Immunohistochemical and clinical characterization of the basal-like subtype of invasive breast carcinoma. Clin Cancer Res 2004;10:5367-5374.

35 Marchio C, Iravani M, Natrajan R, et al. Genomic and immunophenotypical characterization of pure micropapillary carcinomas of the breast. J Pathol 2008;215:398-410.

36 Sekine S, Shibata T, Sakamoto M, et al. Target disruption of the mutant beta-catenin gene in colon cancer cell line HCT116: preservation of its malignant phenotype. Oncogene 2002;21:5906-5911.

37 Parry S, Savage K, Marchio C, et al. Nestin is expressed in basal-like and triple negative breast cancers. J Clin Pathol 2008;61:1045-1050.

38 Savage K, Lambros MB, Robertson D, et al. Caveolin 1 is overexpressed and amplified in a subset of basal-like and metaplastic breast carcinomas: a morphologic, ultrastructural, immunohistochemical, and in situ hybridization analysis. Clin Cancer Res 2007;13: 90-101.
39 Savage K, Leung S, Todd SK, et al. Distribution and significance of caveolin 2 expression in normal breast and invasive breast cancer: an immunofluorescence and immunohistochemical analysis. Breast Cancer Res Treat 2008;110:245-256.

40 Subhawong AP, Subhawong $\mathrm{T}$, Nassar $\mathrm{H}$, et al. Most basal-like breast carcinomas demonstrate the same Rb-/p16+ immunophenotype as the HPV-related poorly differentiated squamous cell carcinomas which they resemble morphologically. Am J Surg Pathol 2009;33:163-175.

41 Rakha EA, El-Sayed ME, Reis-Filho J, et al. Patho-biological aspects of basal-like breast cancer. Breast Cancer Res Treat 2009;113:411-422.

42 Reis-Filho JS, Milanezi F, Steele D, et al. Metaplastic breast carcinomas are basal-like tumours. Histopathology 2006;49:10-21.

43 Weigelt B, Horlings HM, Kreike B, et al. Refinement of breast cancer classification by molecular characterization of histological special types. J Pathol 2008; 216:141-150.

44 Weigelt B, Kreike B, Reis-Filho JS. Metaplastic breast carcinomas are basal-like breast cancers: a genomic profiling analysis. Breast Cancer Res Treat 2009;117:273-280.

45 Bukholm IK, Nesland JM, Borresen-Dale AL. Re-expression of E-cadherin, alpha-catenin and betacatenin, but not of gamma-catenin, in metastatic tissue from breast cancer patients [see comments]. J Pathol 2000;190:15-19.

46 Fodde R, Tomlinson I. Nuclear beta-catenin expression and Wnt signalling: in defence of the dogma. J Pathol 2010;221:239-241.

47 Segditsas S, Rowan AJ, Howarth K, et al. APC and the three-hit hypothesis. Oncogene 2009;28:146-155.

48 Natrajan R, Lambros MB, Rodriguez-Pinilla SM, et al. Tiling path genomic profiling of grade 3 invasive ductal breast cancers. Clin Cancer Res 2009;15: 2711-2722.

49 Natrajan R, Weigelt B, Mackay A, et al. An integrative genomic and transcriptomic analysis reveals molecular pathways and networks regulated by copy number aberrations in basal-like, HER2 and luminal cancers. Breast Cancer Res Treat 2010;121:575-589.

50 Sarrio D, Rodriguez-Pinilla SM, Hardisson D, et al. Epithelial-mesenchymal transition in breast cancer relates to the basal-like phenotype. Cancer Res 2008;68:989-997.

51 Lien HC, Hsiao YH, Lin YS, et al. Molecular signatures of metaplastic carcinoma of the breast by large-scale transcriptional profiling: identification of genes potentially related to epithelial-mesenchymal transition. Oncogene 2007;26:7859-7871.

Supplementary Information accompanies the paper on Modern Pathology website (http://www.nature.com/ modpathol) 